\title{
OPEN Floodplain soils contamination assessment using the sequential extraction method of heavy metals from past mining activities
}

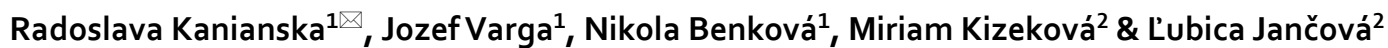

Floodplains are among the most precious and threatened ecosystems in the world. The study deals with floodplain soil contamination caused by 8 heavy metals ( $\mathrm{HMs}$ ) (Cd, $\mathrm{Co}, \mathrm{Cr}, \mathrm{Cu}, \mathrm{Mo}, \mathrm{Ni}, \mathrm{Pb}, \mathrm{Zn}$ ) originating and transported from old mine works along the Štiavnica River in Slovakia. We determined the total HMs content and the HM fractions using BCR sequential extraction method. We selected 12 alluvial sites (AS), two contaminated sites (CS), and one reference site (RS). The sampling points were located within the riparian zones (RZ), arable lands (AL), and grasslands (GL). We confirmed soil contamination by HMs and the related ecological risk by different factors. The contamination by HMs at many AS localities was similar or even higher than at CS localities. The highest contamination factor was calculated for $\mathrm{Cu}$ (39.8), followed by Pb (27.4), Zn (18.2), and $\mathrm{Cd}$ (7.2). The HMs partitioning in the different fractions at the $\mathrm{CS}$ and $\mathrm{AS}$ localities revealed that $\mathrm{Cd}, \mathrm{Zn}$, and $\mathrm{Pb}$ were mainly associated with the exchangeable and reducible fractions, while Cu was mainly associated with the oxidisable fraction. The soil properties were selectively correlated with the HM fractions. Based on the ANOVA results, the effect of different ecosystem types on HM fractions was revealed.

Floodplains are relatively flat land surfaces created when alluvial material carried by surface water is deposited. Rivers and floodplains are connected, exchanging water, sediments, biota, and nutrients in a shared natural ecosystem. In the natural state, floodplains are characterised by high biodiversity with the presence of riparian areas, natural or artificial grasslands, and agricultural land. Floodplains fulfil many ecosystem services, including regulating such as filtration. They cover $7 \%$ of the total European area and host $12 \%$ of the European population. In Slovakia, more than $25 \%$ of the population lives in floodplain areas ${ }^{1}$. Floodplains are among the most dynamic, productive, diverse, and precious ecosystems in the world ${ }^{2,3}$. On the other hand, floodplains are the most threatened ecosystems in the world. In degraded floodplains, the quality of ecosystem services is reduced. In Europe and North America, up to $90 \%$ of floodplains are cultivated and, therefore, functionally extinct. In countries with fast growing economies, there is a huge discharge of pollutants into floodplains that are so vulnerable and highly threatened ${ }^{4}$.

Today, many river floodplains are highly contaminated by HMs that exceed standards for soils and sediments and also the filtering capacity of alluvial soils. Pollution is a very prominent and lasting environmental problem around the world, despite the efforts made in recent decades to clean up the environment. Soil contamination in Europe is a great challenge and puts human and environmental health at risk. Soil contamination is mainly located near waste landfills and industrial activities that spread heavy metals (HMs) and other potentially toxic elements. Important anthropogenic sources of HMs are present in former mining activities, foundries, smelters, and other diffuse sources ${ }^{5}$. Historically, the mining industry is one of the oldest documented types of human activity. Mining waste rocks and mining tailings contain chemicals and residues characterised by a high content of HMs. Mining activities as the main source of HMs constitute a real concern around the world, and many researchers study their impact on soil pollution ${ }^{6-9}$. HMs contained in mining waste can be released from the mining field into the environment and can be dispersed by gravitational transport and fluvial pathways. Floods are directly involved as serious agents of contaminant dispersion ${ }^{10,11}$, resulting in sedimentation on lands, where contaminants can remain for decades and centuries until they are remobilised by surface or river bank erosion ${ }^{12}$.

${ }^{1}$ Faculty of Natural Sciences, Matej Bel University Banská Bystrica, Tajovského 40, 97401 Banská Bystrica, Slovakia. ${ }^{2}$ National Agricultural and Food Centre, Grassland and Mountain Agriculture Research Institute, Mládežnícka 36, 97421 Banská Bystrica, Slovakia. ${ }^{\circledR}$ email: radoslava.kanianska@umb.sk 
In floodplain ecosystems, soils provide important filtration services. Soils remove different contaminants, including HMs from water and prevent their bioavailability to plants and animals. Nevertheless, floodplain soils still have received little attention and their potential contamination is often underestimated or ignored. Little attention is also devoted to a deeper analysis of ecosystem services based on analytical work. The reason is that traditional floodplain management has emphasized the conversion of floodplains to uses such as agriculture, housing, and industry ${ }^{13}$. This emphasis on management has also had enormous economic benefits through the development of intensive agriculture on rich alluvial soils neglecting the real environmental problems and the need to protect soils and their services.

Contaminated floodplain soils and sediments pose a potential danger to the safety of agricultural products and human health, and can adversely affect the environment and ecosystems ${ }^{14}$. There are different studies around the world that focus on contaminated floodplains ${ }^{15-17}$. The large variation in contaminants, soil properties, and other natural and land use conditions at floodplains makes it impossible to assess the full extent of soil pollution and related filtration ecosystem services (FES). In addition, different contaminants have different effects on ecosystems, living organisms, human health, and the environment depending on their properties. The effect of the contaminant depends not only on its quantity, but also on its dispersion potential, solubility, and bioavailability. The total HMs content of soils is useful for many geochemical applications, but the fractions of these metals are often of greater interest. Different fractions exert different environmental effects, directly affecting the toxicity of metals, their migration, and natural cycling ${ }^{18}$. We suppose that the determination of different HM fractions in the soil together with the analysis of the relations between HM fractions and soil properties is possible to assess the FESs and their related capacity.

Therefore, regional studies can be a valuable contribution to a complex mosaic of knowledge about soil pollution and soil FESs. Understanding the identification of heavy metal forms and their interactions with the physical, chemical, and biological properties of soil is the necessary knowledge for a more proper and sustainable ecosystem-based management of floodplains.

The aim of this study is to investigate the contamination of the Štiavnica River floodplain by HMs originated from past mining activities, and: (1) to quantify the total content and the various fractions of HMs in soils with an emphasis on mobile and bioavailable fractions; (2) to assess the environmental impact of HMs and the degree of soil pollution with various factors; (3) to determine the relationships between different soil properties and fractions of HMs that affect the FES capacity of the floodplain ecosystem.

\section{Material and methods}

Sampling sites and soil sampling. Sampling sites were selected in the floodplain along the Štiavnica River in central Slovakia. The riverbanks consist of fluvial deposits (sand and silt). The Štiavnica River is $55 \mathrm{~km}$ long, with a $443 \mathrm{~km}^{2}$ large catchment area. In the upstream part of the basin in the Štiavnické vrchy Mounatins of volcanogenic origin, the relief is dominated by hills and valleys with forests and partly by permanent grasslands used as meadows. The bedrock consists mainly of andesites and rhyolites with scattered occurrences of conglomerates and shales. In the downstream part of the basin in the Podunajska pahorkatina Hills, the relief is dominated by a flat surface. The bedrock consists of clay- and sand-stones on slopes, sand and gravel in areas of floodplains. The land is used as arable land. Due to volcanogenic origin, the region of the Štiavnické vrchy Mountains is mineral-rich. The extraction of precious metals in the central part of the Štiavnické vrchy Mountains has a very long history. Large-scale mining started in the twelfth century. The discovery of precious metals encouraged rapid expansion. Silver ore prevailed among the mined metals. In the eighteenth century, the region became the biggest mining centre in the Habsburg Monarchy. In the period between 1790 and 1863, the mountains yielded 490 metric tons of silver and 11 tons of gold. At that time, there was no environmental legislation preventing the release of contaminated water and sediments into watercourses. At the end of the nineteenth century, mining fell in decay. This trend also continued in the twentieth century. Past mining activities left imprint on the environment, including contamination by HMs and other potentially toxic elements. Nowadays, the storage and handling of tailings is a major environmental issue in the region. There exist several old mine tailings, waste heaps, and dumps. The ore field is drained by the Štiavnica River.

To study the contamination of floodplain soils by HMs from past mining activities and transported by the Štiavnica River and its sediments, we selected a total of 15 study sites along the Štiavnica River, including 2 contaminated sites (2CS, 4CS), 1 reference site (7RS) and 8 alluvial sites (AS) located in the Štiavnické vrchy Mountains (1AS, 3AS, 5AS, 6AS, 8-11 AS), and 4 alluvial sites situated in the Podunajská pahorkatina Hills (12-15 AS) (Fig. 1).

The soil types on the floodplain were classified as Haplic Fluvisols according to the World Reference Base for Soil Resources. The sampling points were located on the river bank within the riparian zones (RZ) in the Štiavnické vrchy Mountains, and on arable land (AL) in the Podunajská pahorkatina Hills. In the case of a larger floodplain, we also collected samples distant from the water flow at the end of the floodplain, that were used as permanent grasslands (GL) in the Štiavnické vrchy Mountains, and as arable land in the Podunajská pahorkatina Hills (at 5 of 12 alluvial sites). Outside the river floodplain, we selected the two contaminated sites (CS) classified as Ekranic Technosols, which are poorly developed on metallurgical slag and shallow soils. As CS sites, we selected the area of the Maximilian shaft that is registered in the Register of environmental burdens of the Slovak republic as probable environmental burden (PEB), and the hard rock mine tailing Lintich that is registered as a confirmed environmental burden (CEB), where hazardous substances caused by mining activities can pose a risk to human health or to the environment, soil and groundwater. The Maximilian shaft area was located approximately $1 \mathrm{~km}$ and the Lintich mine tailing approximately $100 \mathrm{~m}$ from the Štiavnica River. The RS site represents a site with a 'normal' level of contaminants, and arising from a combination of natural and diffuse pollution contributions. The RS site was used to determine the background values. The RS site was located 

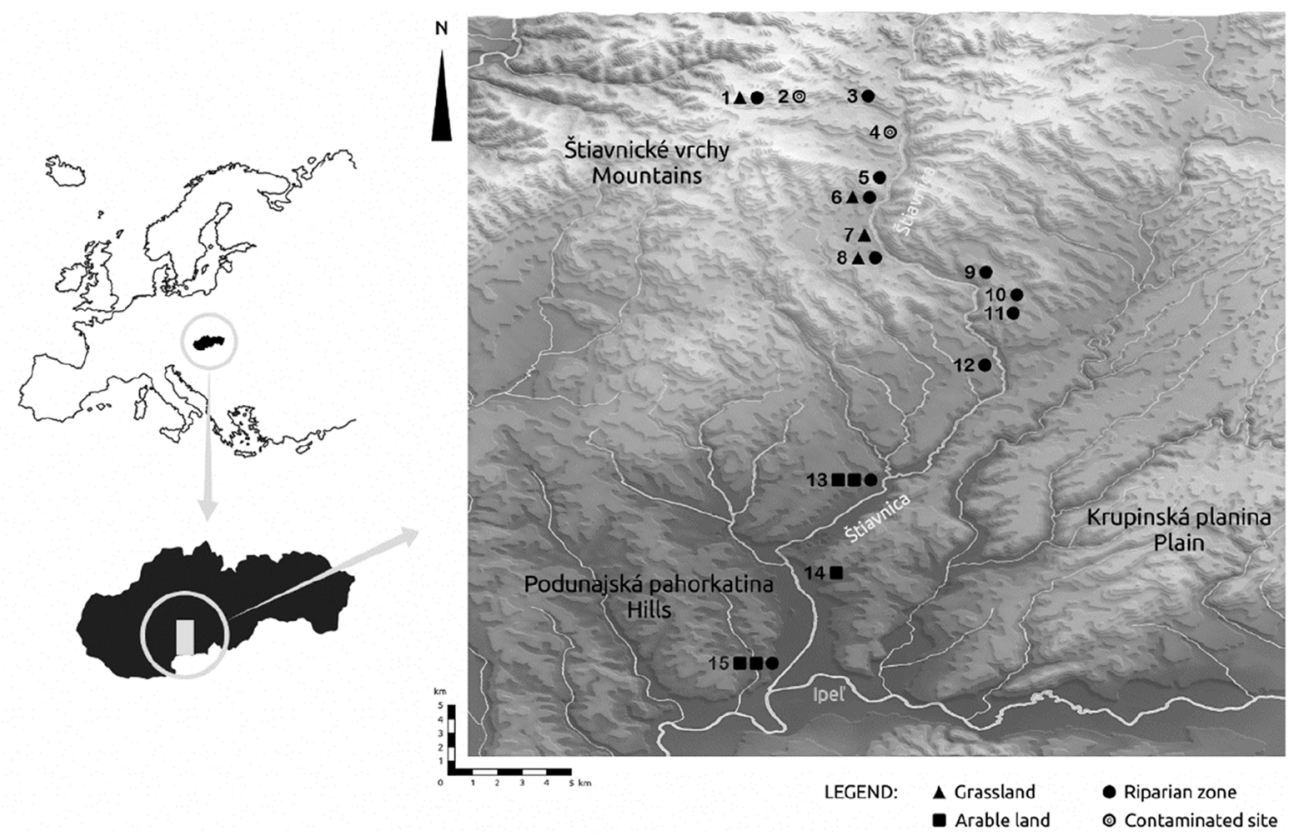

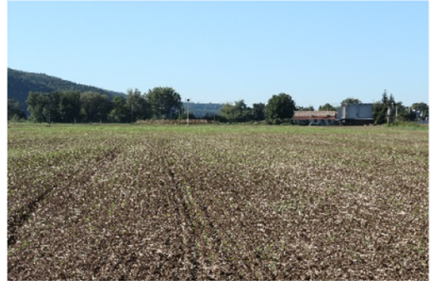

Alluvial site - arable land (13AS-AL)

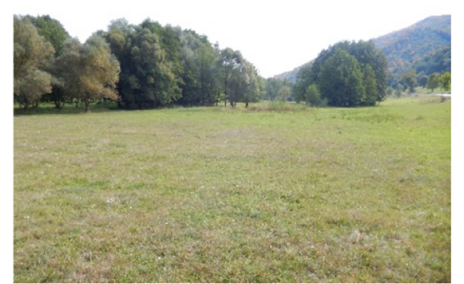

Alluvial site - grassland (8ASGL)

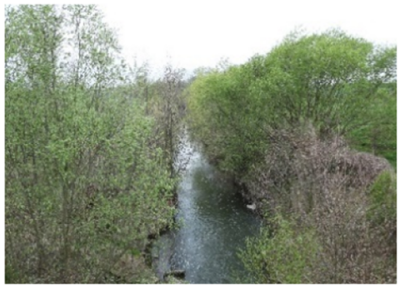

Alluvial site - riparian zone (15AS-RZ)

Figure 1. Isometric map of the sampling points and photos from selected study sites (photos R. Kanianska). The map was generated using 3D Map Generator, ver. 1.5, https://www.3d-map-generator.com/3d-map-gener ator-terrain/.

about $400 \mathrm{~m}$ away from the Štiavnica River outside the floodplain on a slope, and is used as a meadow. The soil type was classified as Haplic Cambisol.

Soil analysis. Soil samples were collected in September 2018 from a depth of 0-10 cm, and 20-30 cm (total of 123), according to the soil profile (presence of bedrock). For the total content of $8 \mathrm{HMs}(\mathrm{Cd}, \mathrm{Co}, \mathrm{Cr}, \mathrm{Cu}, \mathrm{Mo}$, $\mathrm{Ni}, \mathrm{Pb}, \mathrm{Zn}$ ) in the soil was used as the digestion medium the mixture of $\mathrm{HNO}_{3} / \mathrm{HF} / \mathrm{HClO}_{4}$. Then, we applied a three-step sequential extraction scheme introduced by the European Commission through the BCR Programme called the Community Bureau of Reference (BCR) as described by Rauret et al. ${ }^{19}$. The three-steps sequential extraction method operationally defines metals in four chemical forms: (I) Exchangeable and weak acid soluble fraction (HOAc extractable fraction), (II) Reducible fraction (bound to Fe and Mn oxides, extraction with $\mathrm{NH}_{2} \mathrm{OH}-\mathrm{HCl}$ ), (III) Oxidisable fraction (bound to organic matter and sulphides, digestion with $\mathrm{H}_{2} \mathrm{O}_{2}$ was followed by extraction with $\mathrm{NH}_{4} \mathrm{OAc}$ ), and (IV) Residual fraction (bound to silicate minerals). The residual fraction was calculated as the difference between the total content of HM and the sum of the three metal fractions. The procedure followed was inductive coupled plasma optical emission spectrometry (ICP-OES). To guarantee data quality, reagent blanks were used. We measured the Eh in the soil using the Oxygen Diffusion meter (Ejkelkamp). We further determined soil chemical properties such as $\mathrm{pH}$ in $\mathrm{H}_{2} \mathrm{O}$ (with a ratio of 1:2.5) and $1 \mathrm{M}$ $\mathrm{KCl}$ solution (with a ratio of 1:2.5), total soil organic carbon (SOC) using the oxidimetric method according to Tyurin that is similar to the Walkley-Black oxidation method. The Tyurin titrimetric method is a wet combustion method, where soil organic matter is oxidized by $0.2 \mathrm{M} \mathrm{K}_{2} \mathrm{Cr}_{2} \mathrm{O}_{7}$ with $\mathrm{H}_{2} \mathrm{SO}_{4}$. After oxidation, excess dichromate is determined by titration with a Mohr's salt solution. The content of humic and fulvic acids was determined by the short fractionation method according to Kononova and Belchikova using extraction with 
$0.1 \mathrm{M} \mathrm{Na}_{4} \mathrm{P}_{2} \mathrm{O}_{7} \cdot 10 \mathrm{H}_{2} \mathrm{O}$. Oxalate-extractable $\mathrm{Al}, \mathrm{Fe}$ and $\mathrm{Mn}$ as an indicator of hydrous oxides were determined by the acid oxalate extraction method described by Pansu and Gautheyrou ${ }^{20}$ with a $\left(\mathrm{NH}_{4}\right)_{2}$-oxalate solution adjusted to $\mathrm{pH} 3$, shake for $4 \mathrm{~h}$ in the dark and centrifuge for $10 \mathrm{~min}$ at $10,000 \times \mathrm{g}$.

The hydrological soil properties were determined using overall undisturbed soil samples using core-extracting tubes (Eijkelkamp Equipment for Soil Research, The Netherlands). Undisturbed soil samples in tubes were taken to the laboratory for hydrophysical analyses by the gravimetric method of particle density (PD), bulk density (BD), maximum capillary water capacity (MCWC), field water capacity (FWC), actual soil moisture (SM), porosity (as total-Pt, capillary-Pc, non-capillary-Pn, semi-capillary-Ps), and minimal air capacity (MAC) according to Novak. Particle size analysis was performed by pipette method using soil particle sedimentation and particle size fractions (sand, silt, clay) were classified according to the United States Department of Agriculture (USDA) system.

Data analysis. The degree of the soil pollution and the related ecological risk we described with the help of contamination factor (CF), pollution load index (PLI), potential ecological risk of individual metals (Er), and potential ecological risk of the environment (RI).

The CF also designated as an enrichment factor was calculated according to the formula:

$$
\mathrm{CF}=\frac{\mathrm{Ci}}{\mathrm{Cb}}
$$

where $\mathrm{Ci}$ is the measured content of $\mathrm{HM}$ in soil, and $\mathrm{Cb}$ is the background value of the HM contents of the reference site. According to Varol ${ }^{21}$, the $\mathrm{CF}<1$ means low contamination, 1-3 is moderate contamination, 3-6 is considerable contamination, and $\mathrm{CF}>6$ means very high contamination.

To identify heavy metal contamination as a total scale in soil, Tomlinson et al. ${ }^{22}$ recommended an equation for calculating the pollution load index (PLI):

$$
\mathrm{PLI}=\sqrt[\mathrm{n}]{\mathrm{CF} 1 * \mathrm{CF} 2 * \mathrm{CF} 3 \ldots \mathrm{Cfn}}
$$

where $\mathrm{n}$ is the number of metals. PLI $>1$ indicates pollution and demonstrates dynamic deterioration of quality.

According to Hakanson's ${ }^{23}$ methodology, the potential ecological risk (Er) of a given heavy metal is defined as follows:

$$
\operatorname{Er}^{\mathrm{i}}=\operatorname{Tr}^{\mathrm{i}} * \mathrm{CF}^{\mathrm{i}}
$$

where $\operatorname{Tr}$ is the toxic response factor for a given substance, $\mathrm{CF}$ is the contamination factor. The $\operatorname{Tr}$ was suggested by Hakanson ${ }^{23}$ for six metals $\mathrm{Cd}(30), \mathrm{Pb}(5), \mathrm{Hg}(40), \mathrm{Cu}(5), \mathrm{Ni}(5), \mathrm{Zn}(1)$. The sum of individual potential risks $(\mathrm{Er})$ is the potential ecological risk of the environment (RI) of the study site:

$$
\mathrm{RI}=\sum \operatorname{Er}^{\mathrm{i}}
$$

The Er $<40$ means low risk, $40-80$ is moderate risk, $80-160$ is considerable risk, $160-320$ is high risk, and $>320$ is very high risk. The RI $<150$ means low risk, 150-300 is moderate risk, 300-600 is considerable risk, and $>600$ is very high risk.

To identify the relationships between HMs content and soil parameters, statistical analyzes were conducted using the Pearson correlation coefficient. Hierarchical cluster analysis (HCA) was applied to group similar localities (spatial variability) using the Ward's method and Euclidean distances. A two-way ANOVA analysis was performed to assess statistically significant differences in HM fractions between different types of ecosystems (riparian zone, alluvial grassland, arable land), and soil depths $(0-10,20-30 \mathrm{~cm})$. The SPSS Statistics 19 software was used to perform statistical analysis of the data.

\section{Results}

Basic characteristics of soils. The basic statistical characteristics of the chemical and physical properties of the soils were tested in the laboratory and calculated at the CS, AS, and RS sites in two depths. Data are reported in Tables 1, 2. There are differences between the observed properties at specific sites (CS, AS, RS) and in different depths, but also between AS sites themselves, confirming their heterogeneity.

Soil contamination by heavy metals. The total HM content in the two soil depths of the Štiavnica River floodplain together with the Slovak, Finish and Canadian guideline values of the contaminated soil are presented in Table 3. The Finish legislation sets content levels for each hazardous element to identify soil contamination and remediation needs. It distinguishes the so-called 'threshold value' and 'guideline value'. If the guideline value is exceeded, the area has a contamination level that presents ecological or health risks.

For all $\mathrm{HMs}$, except $\mathrm{Cr}$ and $\mathrm{Ni}$, we recorded at least one locality exceeding one of the limit values. In the case of Mo, the total content exceeded the limit value only at one CS locality. The total Co content slightly exceeded only the Slovak limit value at CS localities and several AS localities, and was below other national limit values. We recorded $4 \mathrm{HMs}(\mathrm{Cd}, \mathrm{Cu}, \mathrm{Pb}, \mathrm{Zn}$ ) whose total content in most localities significantly exceeded all limit values, including the Finnish guideline values for soils with decontamination and remediation needs. At AS localities, the situation in total HM content of both observed soil depths was similar. HM contamination was also observed across broader floodplains. 


\begin{tabular}{|c|c|c|c|c|c|c|c|c|c|c|}
\hline Depth $(\mathrm{cm})$ & Site & & $\mathrm{pH} / \mathrm{H}_{2} \mathrm{O}$ & $\mathrm{pH} / \mathrm{KCl}$ & Eh $(\mathrm{mV})$ & SOC $(\mathrm{g} / \mathrm{kg})$ & HA/FA & $\mathrm{Al}_{\mathrm{o}}(\mathrm{mg} / \mathrm{kg})$ & $\mathrm{Fe}_{\mathrm{o}}(\mathrm{mg} / \mathrm{kg})$ & $\mathrm{Mn}_{\mathrm{o}}(\mathrm{mg} / \mathrm{kg})$ \\
\hline \multirow{9}{*}{$0-10$} & \multirow{4}{*}{$\begin{array}{l}\mathrm{CS} \\
(\mathrm{n}=2)\end{array}$} & $\overline{\mathrm{x}}$ & 7.5 & 7.2 & n.a. & 24.30 & 0.57 & 1373.8 & $11,398.5$ & 4817.9 \\
\hline & & SD & 0.1 & 0.2 & n.a. & 9.30 & 0.02 & 573.4 & 4312.0 & 3708.5 \\
\hline & & Min & 7.4 & 7.0 & n.a. & 15.00 & 0.55 & 800.4 & 7086.6 & 1109.4 \\
\hline & & Max & 7.6 & 7.4 & n.a. & 33.60 & 0.58 & 1947.2 & $15,710.5$ & 8526.3 \\
\hline & \multirow{4}{*}{$\begin{array}{l}\text { AS } \\
(\mathrm{n}=19)\end{array}$} & $\overline{\mathrm{x}}$ & 6.3 & 5.5 & 351.95 & 23.08 & 0.43 & 1327.0 & 8280.2 & 2838.4 \\
\hline & & SD & 0.6 & 0.8 & 151.80 & 6.84 & 0.07 & 403.9 & 1338.7 & 2774.8 \\
\hline & & Min & 5.4 & 4.4 & -274.00 & 11.25 & 0.31 & 931.7 & 6255.8 & 622.0 \\
\hline & & Max & 7.7 & 7.2 & 484.00 & 36.60 & 0.59 & 2164.2 & $10,933.5$ & $10,829.5$ \\
\hline & RS & $\mathrm{x}$ & 5.7 & 4.6 & 364.00 & 34.95 & 0.32 & 2625.7 & 2876.4 & 740.5 \\
\hline \multirow{5}{*}{$20-30$} & \multirow{4}{*}{$\begin{array}{l}\text { AS } \\
(\mathrm{n}=12)\end{array}$} & $\overline{\mathrm{x}}$ & 6.3 & 5.4 & n.a. & 16.95 & 0.44 & 1478. & $10,364.3$ & 3285.5 \\
\hline & & SD & 0.8 & 1.0 & n.a. & 6.02 & 0.11 & 436.4 & 2733.4 & 3758.3 \\
\hline & & Min & 5.0 & 4.1 & n.a. & 6.75 & 0.32 & 941.8 & 6855.4 & 628.8 \\
\hline & & Max & 7.6 & 7.1 & n.a. & 32.25 & 0.68 & 2496.5 & $15,455.4$ & $13,448.7$ \\
\hline & RS & $\mathrm{x}$ & 5.8 & 4.7 & n.a. & 22.50 & 0.37 & \begin{tabular}{|l|}
2914.4 \\
\end{tabular} & 3518.0 & 991.9 \\
\hline
\end{tabular}

Table 1. Soil chemical properties in the soil depth of $0-10$ and $20-30 \mathrm{~cm}$ in contaminated, alluvial and reference sites with basic statistical characteristics. $A S$ alluvial site, $C S$ contaminated site, $R S$ reference site, $。$ extracted by oxalate.

\begin{tabular}{|c|c|c|c|c|c|c|c|c|c|c|c|c|c|c|c|}
\hline \multirow[b]{2}{*}{ Depth (cm) } & \multirow[b]{2}{*}{ Site } & & \multicolumn{3}{|c|}{$\begin{array}{l}\text { Content of size } \\
\text { particles \% }\end{array}$} & \multirow[b]{2}{*}{$\mathrm{PD}\left(\mathrm{g} / \mathrm{cm}^{3}\right)$} & \multirow[b]{2}{*}{$\mathrm{BD}\left(\mathrm{g} / \mathrm{cm}^{3}\right)$} & \multirow[b]{2}{*}{ MCWC (\%) } & \multirow[b]{2}{*}{ FWC (\%) } & \multirow[b]{2}{*}{ SM (\%) } & \multirow[b]{2}{*}{ Pt (\%) } & \multirow[b]{2}{*}{ Pc (\%) } & \multirow[b]{2}{*}{ Pn (\%) } & \multirow[b]{2}{*}{ Ps (\%) } & \multirow[b]{2}{*}{ MAC (\%) } \\
\hline & & & Clay & \begin{tabular}{|l|} 
Silt \\
\end{tabular} & Sand & & & & & & & & & & \\
\hline \multirow{9}{*}{$0-10$} & \multirow{4}{*}{$\begin{array}{l}\mathrm{CS} \\
(\mathrm{n}=2)\end{array}$} & $\overline{\mathrm{x}}$ & 7.59 & 36.95 & 55.46 & 2.33 & 1.26 & 23.75 & 20.35 & 19.86 & 45.83 & 20.35 & 20.40 & 5.08 & 22.08 \\
\hline & & SD & 0.30 & 5.14 & 5.44 & 0.10 & 0.08 & 0.57 & 0.74 & 0.54 & 1.06 & 0.74 & 1.41 & 0.39 & 1.63 \\
\hline & & Min & 7.29 & 31.81 & 50.02 & 2.23 & 1.18 & \begin{tabular}{|l|}
23.17 \\
\end{tabular} & 19.60 & 19.32 & 44.77 & \begin{tabular}{|l|}
19.60 \\
\end{tabular} & 18.99 & 4.69 & 20.45 \\
\hline & & Max & 7.89 & 42.09 & 60.90 & 2.43 & 1.34 & 24.32 & 21.09 & 20.40 & 46.89 & 21.09 & 21.82 & 5.47 & 23.72 \\
\hline & \multirow{4}{*}{$\begin{array}{l}\text { AS } \\
(\mathrm{n}=19)\end{array}$} & $\overline{\mathrm{x}}$ & 10.22 & 47.54 & \begin{tabular}{|l|}
42.18 \\
\end{tabular} & 2.25 & 1.10 & 36.99 & 32.37 & 30.17 & 51.36 & 32.37 & 13.56 & 5.43 & 14.37 \\
\hline & & SD & 8.05 & 16.58 & 20.63 & 0.14 & 0.21 & 8.83 & 8.68 & 12.12 & 8.48 & 8.68 & 13.19 & 5.29 & 12.36 \\
\hline & & Min & 4.45 & 19.05 & 4.32 & 1.91 & 0.72 & 22.30 & 20.26 & 15.68 & 35.59 & 35.59 & 20.26 & 0.00 & 0.00 \\
\hline & & $\operatorname{Max}$ & 42.28 & 77.76 & 76.62 & 2.59 & 1.50 & 57.20 & 51.65 & 57.09 & 66.16 & 66.16 & 51.65 & 39.37 & 46.66 \\
\hline & RS & $\mathrm{x}$ & 25.81 & 51.48 & 22.71 & 2.43 & 1.34 & 34.70 & 32.28 & 19.96 & 36.29 & 32.28 & 0.17 & 3.84 & 1.59 \\
\hline \multirow{4}{*}{$20-30$} & \multirow{4}{*}{$\begin{array}{l}\text { AS } \\
(\mathrm{n}=12)\end{array}$} & $\overline{\mathrm{x}}$ & 10.63 & 40.00 & 49.38 & 2.26 & 1.19 & 36.95 & 31.98 & 29.03 & 46.82 & 31.98 & 8.51 & 6.33 & 9.87 \\
\hline & & SD & 9.12 & 15.63 & 21.16 & 0.19 & 0.28 & 8.96 & 11.07 & 13.45 & 13.40 & 11.07 & 10.31 & 5.09 & 10.31 \\
\hline & & Min & 4.35 & 22.78 & \begin{tabular}{|l|}
8.53 \\
\end{tabular} & \begin{tabular}{|l|}
2.04 \\
\end{tabular} & \begin{tabular}{|l|}
0.69 \\
\end{tabular} & 30.35 & 18.67 & 10.88 & 30.95 & \begin{tabular}{|l|}
18.67 \\
\end{tabular} & 0.00 & 0.23 & 0.00 \\
\hline & & Max & 39.43 & \begin{tabular}{|l|l}
78.81 \\
\end{tabular} & \begin{tabular}{|l|l}
72.87 \\
\end{tabular} & \begin{tabular}{|l|}
2.67 \\
\end{tabular} & \begin{tabular}{|l}
1.54 \\
\end{tabular} & 58.37 & 56.44 & 58.12 & 67.47 & 56.44 & 24.72 & \begin{tabular}{|l|}
17.28 \\
\end{tabular} & 27.14 \\
\hline
\end{tabular}

Table 2. Soil physical properties in the soil depth of $0-10$ and $20-30 \mathrm{~cm}$ in contaminated, alluvial and reference sites with basic statistical characteristics. $A S$ alluvial site, $C S$ contaminated site, $R S$ reference site.

Contamination and ecological risk assessment for heavy metals in soil. Contamination and ecological risk at the CS and AS localities were confirmed by calculated indices along the entire Štiavnica River floodplain including the downstream part of the Podunajská pahorkatina Hills (Table 4).

In contaminated sites, the average $\mathrm{CF}$ showed a very high contamination by $\mathrm{Zn}$ and $\mathrm{Cu}$; considerable contamination by $\mathrm{Cd}, \mathrm{Mo}, \mathrm{Pb}$; and moderate contamination by $\mathrm{Co}, \mathrm{Cr}$, and $\mathrm{Ni}$. At alluvial sites, the average $\mathrm{CF}$ showed very high contamination by $\mathrm{Cu}, \mathrm{Pb}$, and $\mathrm{Zn}$; considerable contamination by $\mathrm{Cd}$; moderate contamination by $\mathrm{Cr}$, $\mathrm{Mo}$, and $\mathrm{Ni}$; and low contamination by $\mathrm{Co}$. Very high contamination $(\mathrm{CF}>6)$ was calculated for $\mathrm{Pb}, \mathrm{Zn}$, and $\mathrm{Cu}$ at most AS localities. Overall, the highest $\mathrm{CF}$ was calculated in the case of $\mathrm{Cu}$ (39.8 in the 15AS-AL locality), $\mathrm{Pb}$ (27.4 in the 1AS-RZ locality), $\mathrm{Zn}$ (18.2 in the 6AS-GL locality), Cd (7.2 in the 1AS-GL locality). Contamination by HMs was, in many AS localities, similar or even higher than in CS localities. Metal PLI values confirmed that the CS and AS sites were contaminated except for five AS localities, two of them are used as grasslands, two as arable land, and one is located in the riparian zone. The average Er showed a considerable risk of Cd at the AS and CS localities, and moderate risk of $\mathrm{Cu}$ and $\mathrm{Pb}$ at the AS localities. The RI values indicated a considerable ecological and environmental risk at 7 study sites located mainly in riparian forests, moderate risk at 11 study sites and only 2 study sites located in the riparian zone were at low risk.

Heavy metals fractionation. The sequential extraction procedure enables us to measure broader forms or phases. 


\begin{tabular}{|c|c|c|c|c|c|c|c|c|c|c|}
\hline Depth (cm) & Site & Locality & $\mathrm{Cd}$ & Co & $\mathrm{Cr}$ & $\mathrm{Cu}$ & Mo & $\mathrm{Ni}$ & $\mathrm{Pb}$ & $\mathrm{Zn}$ \\
\hline \multirow{28}{*}{$0-10$} & \multirow{4}{*}{ CS } & 2CS-PEB & 13.18 & 16.90 & 32.72 & 90.18 & 12.28 & 27.83 & 868.39 & 1841.51 \\
\hline & & 4CS-CEB & 6.96 & 17.89 & 33.39 & 81.80 & 2.03 & 22.74 & \begin{tabular}{|l|}
2924.80 \\
\end{tabular} & 979.39 \\
\hline & & $\bar{x}$ & 10.07 & 17.40 & 33.05 & 85.99 & 7.15 & 25.29 & \begin{tabular}{|l|}
1896.59 \\
\end{tabular} & 1410.45 \\
\hline & & $S D$ & 3.11 & 0.49 & 0.33 & 4.19 & 5.13 & 2.55 & 1028.21 & 431.06 \\
\hline & \multirow{23}{*}{ AS } & 1AS-RZ & 14.16 & 15.68 & 24.35 & 122.32 & 3.83 & 18.02 & 2602.79 & 1948.56 \\
\hline & & 1AS-GL & 23.95 & 7.98 & 11.98 & 143.71 & 3.99 & 11.98 & 890.22 & 2155.69 \\
\hline & & 3AS-RZ & 12.14 & 11.56 & 19.58 & 117.68 & 3.08 & 12.95 & 631.60 & 1566.59 \\
\hline & & 5AS-RZ & 3.93 & 16.62 & 29.40 & 35.26 & 1.03 & 17.86 & 401.56 & 454.68 \\
\hline & & 6AS-RZ & 15.85 & 17.69 & 25.97 & 252.70 & 1.86 & 15.94 & \begin{tabular}{|l|}
919.51 \\
\end{tabular} & 2032.25 \\
\hline & & 6AS-GL & 11.97 & 11.97 & 23.93 & 227.36 & 0.00 & 19.94 & \begin{tabular}{|l|}
1108.90 \\
\end{tabular} & 3103.31 \\
\hline & & 8AS-RZ & 14.65 & 17.56 & 24.97 & 156.93 & 2.29 & 17.53 & 602.72 & 1920.56 \\
\hline & & 8AS-GL & 3.94 & 11.81 & 23.62 & 35.43 & 0.00 & 11.81 & 153.54 & 307.09 \\
\hline & & 9AS-RZ & 19.57 & 13.69 & 20.10 & 235.96 & 3.46 & 13.53 & \begin{tabular}{|l|}
1245.83 \\
\end{tabular} & 2044.74 \\
\hline & & 10AS-RZ & 11.81 & 17.35 & 22.24 & 210.75 & 2.90 & 13.86 & \begin{tabular}{|l|}
783.27 \\
\end{tabular} & 1327.99 \\
\hline & & 11AS-RZ & 16.39 & 15.40 & 18.46 & 266.59 & 2.35 & 13.11 & 1042.77 & 1810.83 \\
\hline & & 12AS-RZ & 16.06 & 13.44 & 19.26 & 222.32 & 1.44 & 12.45 & 1357.63 & 1669.09 \\
\hline & & 13AS-RZ & 14.06 & 18.09 & 34.05 & 147.01 & 2.66 & 18.46 & 977.43 & 1362.35 \\
\hline & & 13AS-AL-1 & 11.84 & 11.84 & 35.53 & 126.33 & 0.00 & 15.79 & 1508.09 & 1267.27 \\
\hline & & 13AS-AL-2 & 11.96 & 15.94 & 51.81 & 151.45 & 0.00 & 27.90 & 1466.72 & 1502.59 \\
\hline & & 14AS-AL & 14.73 & 12.63 & 28.79 & 95.80 & 1.16 & 15.69 & 1512.26 & 1653.78 \\
\hline & & 15AS-RZ & 11.87 & 11.87 & 55.38 & 106.80 & 0.00 & 27.69 & 439.08 & 1079.91 \\
\hline & & 15AS-AL-1 & 23.71 & 15.80 & 43.46 & 537.34 & 3.95 & 23.71 & 2580.01 & 2370.60 \\
\hline & & 15AS-AL-2 & 19.67 & 13.95 & 62.11 & 170.33 & 1.57 & 34.78 & 1104.50 & 2106.45 \\
\hline & & $\bar{x}$ & 14.33 & 14.26 & 30.26 & 176.95 & 1.87 & 18.05 & 1122.55 & 1667.60 \\
\hline & & $S D$ & 5.12 & 2.66 & 13.32 & 106.78 & 1.41 & 6.14 & 627.45 & 628.24 \\
\hline & & $\operatorname{Min}$ & 3.93 & 7.98 & 11.98 & 35.26 & 0.00 & 11.81 & 153.54 & 307.09 \\
\hline & & $\operatorname{Max}$ & 23.95 & 18.09 & 62.11 & 537.34 & 3.99 & 34.78 & 2602.79 & 3103.31 \\
\hline & RS & 7RS-GL & 3.33 & 15.20 & 24.92 & 13.48 & 1.55 & 14.70 & $\begin{array}{l}94.87 \\
\end{array}$ & 170.25 \\
\hline \multirow{17}{*}{$20-30$} & \multirow{16}{*}{ AS } & 1AS-RZ & 14.20 & 13.98 & 21.95 & 127.28 & 3.85 & 14.77 & \begin{tabular}{|l|}
1081.98 \\
\end{tabular} & 1744.37 \\
\hline & & 3AS-RZ & 13.18 & 13.11 & 21.80 & 134.37 & 3.77 & 14.67 & 506.67 & 1710.54 \\
\hline & & 5AS-RZ & 3.48 & 13.69 & 30.03 & 30.42 & 0.72 & 16.44 & 377.30 & 354.14 \\
\hline & & 6AS-RZ & 6.52 & 14.70 & 20.98 & 327.33 & 2.45 & 12.72 & 779.92 & 801.32 \\
\hline & & 8AS-RZ & 10.86 & 17.72 & 21.73 & 144.88 & 1.92 & 15.92 & 607.55 & 1241.98 \\
\hline & & 9AS-RZ & 13.83 & 14.53 & 27.62 & 186.04 & 1.20 & 15.16 & 953.02 & 1769.38 \\
\hline & & 10AS-RZ & 11.28 & 18.86 & 23.40 & 226.91 & 1.95 & 15.04 & \begin{tabular}{|l|}
1032.18 \\
\end{tabular} & 1570.78 \\
\hline & & 11AS-RZ & 14.54 & 15.01 & 22.71 & 238.26 & 2.50 & 13.45 & 995.32 & 1812.79 \\
\hline & & 12AS-RZ & 23.27 & 14.51 & 17.71 & 308.42 & 2.31 & 13.00 & \begin{tabular}{|l|}
1262.08 \\
\end{tabular} & 2033.20 \\
\hline & & 13AS-RZ & 13.61 & 19.13 & 25.86 & 140.62 & 2.31 & 17.43 & 867.61 & 1250.05 \\
\hline & & 14AS-AL & 15.78 & 15.20 & 27.24 & 111.08 & 1.65 & 16.77 & 1425.55 & 1857.01 \\
\hline & & 15AS-AL-2 & 18.03 & 13.29 & 58.91 & 173.34 & 1.23 & 33.86 & 1104.08 & 2131.51 \\
\hline & & $\bar{x}$ & 13.21 & 15.31 & 26.66 & 179.08 & 2.16 & 16.60 & \begin{tabular}{|l|l|}
916.10 \\
\end{tabular} & 1523.09 \\
\hline & & $S D$ & 4.85 & 2.00 & 10.25 & 80.95 & 0.91 & 5.39 & 294.00 & 501.15 \\
\hline & & $\operatorname{Min}$ & 3.48 & 13.11 & 17.71 & 30.42 & 0.72 & 12.72 & 377.30 & 354.14 \\
\hline & & Max & 23.27 & 19.13 & 58.91 & 327.33 & 3.85 & 33.86 & 1425.55 & 2131.51 \\
\hline & RS & 7RS-GL & 3.94 & 11.83 & 23.67 & 23.67 & 0.00 & 11.83 & 110.45 & 177.51 \\
\hline \multirow{5}{*}{ Limits } & & 1 & $0.4-1.0$ & $15-20$ & $100-200$ & $30-70$ & & $40-60$ & $25-115$ & $100-200$ \\
\hline & & 2 & 1 & 20 & 100 & 100 & & 50 & 60 & 200 \\
\hline & & 3 & $10-20$ & $100-250$ & $200-300$ & $150-200$ & & $100-150$ & $200-750$ & $250-400$ \\
\hline & & 4 & 1.4 & 40 & 64 & 63 & 5 & 45 & 70 & 250 \\
\hline & & 5 & 22 & 300 & 87 & 91 & 40 & 89 & 600 & 410 \\
\hline
\end{tabular}

Table 3. Total content ( $\mathrm{T}$ ) of heavy metals in the soil depth of $0-10$ and $20-30 \mathrm{~cm}$ in contaminated, alluvial and reference sites with basic statistical characteristics compared to threshold and guideline values (mg/kg). AS alluvial site, $C S$ contaminated site, $R S$ reference site (used to determine background values), $R Z$ riparian zone, $A L$ arable land, $G L$ grassland, $P E B$ probable environmental burden, $C E B$ confirmed environmental burden. Signs mean: 1-Slovak threshold value of soil contamination in agricultural soil, 2-Finish threshold value of soil contamination, 3-Finish guideline values of soil contamination and remediation needs, 4-Canadian soil quality guidelines for the protection of environmental and human health for agricultural land, 5-Canadian soil quality guidelines for the protection of environmental and human health for industrial land. Normal letter means below all threshold values; bold letter means above at least one threshold value. 


\begin{tabular}{|c|c|c|c|c|c|c|c|c|c|c|c|c|c|c|c|}
\hline \multirow[b]{2}{*}{ Site } & \multirow[b]{2}{*}{ Locality } & \multicolumn{8}{|l|}{$\mathrm{CF}$} & \multirow[b]{2}{*}{ PLI } & \multicolumn{4}{|l|}{ Er } & \multirow[b]{2}{*}{ RI } \\
\hline & & Cd & Co & $\mathrm{Cr}$ & $\mathrm{Cu}$ & Mo & $\mathrm{Ni}$ & $\mathbf{P b}$ & $\mathrm{Zn}$ & & Cd & $\mathrm{Cu}$ & $\mathbf{P b}$ & $\mathrm{Zn}$ & \\
\hline \multirow{3}{*}{ CS } & 2CS-PEB & 4.0 & 1.1 & 1.3 & 6.7 & 7.9 & 1.9 & 4.8 & \begin{tabular}{|l|l|}
10.8 \\
\end{tabular} & 3.1 & 120.0 & 33.5 & 24.0 & 10.8 & 188.3 \\
\hline & 4CS-CEB & 2.1 & 1.2 & 1.3 & 6.1 & 1.3 & 1.5 & 5.5 & 5.8 & 2.0 & 63.0 & 30.5 & 27.5 & 5.8 & 126.8 \\
\hline & $\bar{x}$ & 3.0 & 1.1 & 1.3 & 6.4 & 4.6 & 1.7 & 5.2 & 8.3 & 2.6 & 91.5 & 32.0 & 25.8 & 8.3 & 157.6 \\
\hline \multirow{23}{*}{ AS } & 1AS-RZ & 4.3 & 1.0 & 1.0 & 9.1 & 2.5 & 1.2 & 27.4 & 11.4 & 3.1 & 129.0 & 45.5 & 137.0 & 11.4 & 322.9 \\
\hline & 1AS-GL & 7.2 & 0.5 & 0.5 & 10.7 & 2.6 & 0.8 & 9.4 & 12.7 & 2.4 & 216.0 & 53.5 & 47.0 & 12.7 & 329.2 \\
\hline & 3AS-RZ & 3.6 & 0.8 & 0.8 & 8.7 & 2.0 & 0.9 & 6.7 & 9.2 & 2.2 & 108.0 & 43.5 & 33.5 & 9.2 & 194.2 \\
\hline & 5AS-RZ & 1.2 & 1.1 & 1.2 & 2.6 & 0.7 & 1.2 & 4.2 & 2.7 & 1.4 & 36.0 & 13.0 & 21.0 & 2.7 & 72.7 \\
\hline & 6AS-RZ & 4.8 & 1.2 & 1.0 & 18.7 & 1.2 & 1.1 & 9.7 & 11.9 & 2.9 & 144.0 & 93.5 & 48.5 & 11.9 & 297.9 \\
\hline & 6AS-GL & 3.6 & 0.8 & 1.0 & 16.9 & 0.0 & 1.4 & 11.7 & 18.2 & 0.8 & 108.0 & 84.5 & 58.5 & 18.2 & 269.2 \\
\hline & 8AS-RZ & 4.4 & 1.2 & 1.0 & 11.6 & 1.5 & 1.2 & 6.4 & 11.3 & 2.7 & 132.0 & 58.0 & 32.0 & 11.3 & 233.3 \\
\hline & 8AS-GL & 1.2 & 0.8 & 0.9 & 2.6 & 0.0 & 0.8 & 1.6 & 1.8 & 0.4 & 36.0 & 13.0 & 8.0 & 1.8 & 58.8 \\
\hline & 9AS-RZ & 5.9 & 0.9 & 0.8 & 17.5 & 2.2 & 0.9 & 13.1 & 12.0 & 2.9 & 177.0 & 87.5 & 65.5 & 1.2 & 342.0 \\
\hline & 10AS-RZ & 3.5 & 1.1 & 0.9 & 15.6 & 1.9 & 0.9 & 8.3 & 7.8 & 2.6 & 105.0 & 78.0 & 41.5 & 7.8 & 232.3 \\
\hline & 11AS-RZ & 4.9 & 1.0 & 0.7 & 19.8 & 1.5 & 0.9 & 11.0 & 10.6 & 2.7 & 147.0 & 99.0 & 55.0 & 10.6 & 311.6 \\
\hline & 12AS-RZ & 4.8 & 0.9 & 0.8 & 16.5 & 0.9 & 0.8 & 14.3 & 9.8 & 2.5 & 144.0 & 82.5 & 71.5 & 9.8 & 307.8 \\
\hline & 13AS-RZ & 4.2 & 1.2 & 1.4 & 10.9 & 1.7 & 1.3 & 10.3 & 8.0 & 2.7 & 126.0 & 54.5 & 51.5 & 8.0 & 240.0 \\
\hline & 13AS-AL-1 & 3.6 & 0.8 & 1.4 & 9.4 & 0.0 & 1.1 & 15.9 & 7.4 & 0.7 & 108.0 & 47.0 & 79.5 & 7.4 & 241.9 \\
\hline & 13AS-AL-2 & 3.6 & 1.0 & 2.1 & 11.2 & 0.0 & 1.9 & 15.5 & 8.8 & 0.8 & 108.0 & 56.0 & 77.5 & 8.8 & 250.3 \\
\hline & 14AS_AL & 4.4 & 0.8 & 1.2 & 7.1 & 0.7 & 1.1 & 15.9 & 9.7 & 2.3 & 132.0 & 35.5 & 79.5 & 9.7 & 256.7 \\
\hline & 15AS-RZ & 3.6 & 0.8 & 2.2 & 7.9 & 0.0 & 1.9 & 4.6 & 6.3 & 0.7 & 108.0 & 39.5 & 23.0 & 6.3 & 176.8 \\
\hline & 15AS-AL-1 & 7.1 & 1.0 & 1.7 & 39.8 & 2.5 & 1.6 & 27.2 & 13.9 & 4.1 & 213.0 & 199.0 & 136.0 & 13.9 & 561.9 \\
\hline & 15AS-AL-2 & 5.9 & 0.9 & 2.5 & 12.6 & 1.0 & 2.4 & 11.6 & 12.4 & 3.0 & 177.0 & 63.0 & 58.0 & 12.4 & 310.4 \\
\hline & $\bar{x}$ & 4.3 & 0.9 & 1.2 & 13.1 & 1.2 & 1.2 & 11.8 & 9.8 & 2.2 & 129.2 & 65.6 & 59.2 & 9.8 & 263.7 \\
\hline & $S D$ & 1.5 & 0.18 & 0.5 & 7.9 & 0.9 & 0.4 & 6.6 & 3.7 & 1.0 & 45.9 & 39.6 & 33.0 & 3.7 & 103.7 \\
\hline & Min & 1.2 & 0.5 & 0.5 & 2.6 & 0.0 & 0.8 & 1.6 & 1.8 & 0.4 & 36.0 & 13.0 & 8.0 & 1.8 & 58.8 \\
\hline & Max & 7.2 & 1.2 & 2.5 & 39.8 & 2.6 & 2.4 & 27.4 & 18.2 & 4.1 & 216.0 & 199.0 & 137.0 & 18.2 & 561.9 \\
\hline
\end{tabular}

Table 4. Contamination factor (CF), pollution load index (PLI), potential ecological risk factor (Er), potential ecological risk of the environment (RI) for contaminated and alluvial sites in the soil depth of $0-10 \mathrm{~cm}$. $A S$ alluvial site, $C S$ contaminated site, $R Z$ riparian zone, $A L$ arable land, GL grassland, $P E B$ probable environmental burden, $C E B$ confirmed environmental burden. The $C F<1$ means low contamination, $1-3$ is moderate contamination, 3-6 is considerable contamination and $\mathrm{CF}>6$ means very high contamination. The PLI $>1$ indicates pollution. The Er $<40$ means low risk, $40-80$ is moderate risk, $80-160$ is considerable risk, $160-320$ is high risk, and $>320$ is very high risk. The $\mathrm{RI}<150$ means low risk, $150-300$ is moderate risk, $300-600$ is considerable risk, and $>600$ is very high risk.

HMs were distributed in all four fractions (exchangeable, reducible, oxidisable, and residual) between the CS, AS, and RS localities (Fig. 2a-c).

The content of HMs in the residual fraction was dominated at the RS localities (in 7 of $8 \mathrm{HMs}$ ). The study of the partitioning of HMs in the different fractions at the CS and AS localities revealed that $\mathrm{Cd}, \mathrm{Cu}, \mathrm{Pb}$, and $\mathrm{Zn}$ were mainly associated with the first three fractions. The first exchangeable fraction was the highest in the case of $\mathrm{Cd}$ and $\mathrm{Zn}$. Cu was mainly associated with the oxidisable fraction bound to organic matter and sulphides. The distribution of HMs in the different fractions, mainly in exchangeable ones, suggested that the mobility and bioavailability of the metals probably declined in the order of $\mathrm{Cd}, \mathrm{Zn}, \mathrm{Cu}$, and $\mathrm{Pb}$ at $\mathrm{AS}$ localities, and $\mathrm{Zn}, \mathrm{Cd}$, $\mathrm{Pb}$, and $\mathrm{Cu}$ at $\mathrm{CS}$ localities.

The four most serious fractions of pollutants $(\mathrm{Cd}, \mathrm{Cu}, \mathrm{Pb}, \mathrm{Zn})$ in the Štiavnica River floodplain indicate their different distribution patterns. At AS localities, on average, the content of Cd represented $46.0 \%$ in residual, $28.4 \%$ in exchangeable, $20.9 \%$ in reducible, and $4.9 \%$ in oxidisable fraction. At CS localities, the highest percentages of Cd were associated with the reducible (32.2\%), exchangeable (27.4\%), residual (25.0\%) and oxidisable (15.2\%) fraction.

At AS localities, on average, the Cu content accounted for $49.2 \%$ in oxidisable, $36.8 \%$ in residual, 8.3 in reducible, and $5.7 \%$ in exchangeable fraction. At CS localities, the share of different fractions was similar, but the $\mathrm{Cu}$ share bond on organic matter and sulphides was higher (72.1\%).

At AS localities, on average, the content of $\mathrm{Pb}$ represented $46.7 \%$ in reducible, $37.0 \%$ in residual, 15.3 in oxidisable, and $1.0 \%$ in exchangeable fraction. At CS localities, the share of different fractions was different, $51.5 \%$ in reducible, $32.9 \%$ in oxidisable, $4.2 \%$ in exchangeable, and $11.3 \%$ in residual fraction.

At AS localities, on average, the content of $\mathrm{Zn}$ represented $42.3 \%$ in residual, $23.7 \%$ in reducible, $19.9 \%$ in exchangeable, and $14.1 \%$ in oxidisable fraction. At CS localities, the share of the different fractions was different, $34.4 \%$ in exchangeable, 26.2 in reducible, $25.1 \%$ in oxidisable, and $14.3 \%$ in residual fraction. 
(a)

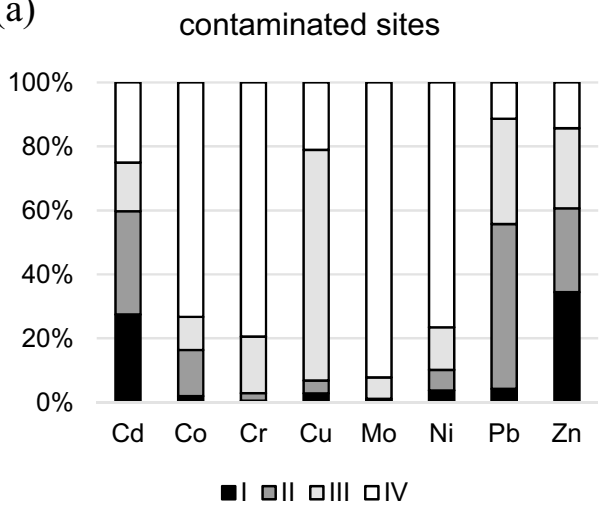

(b)

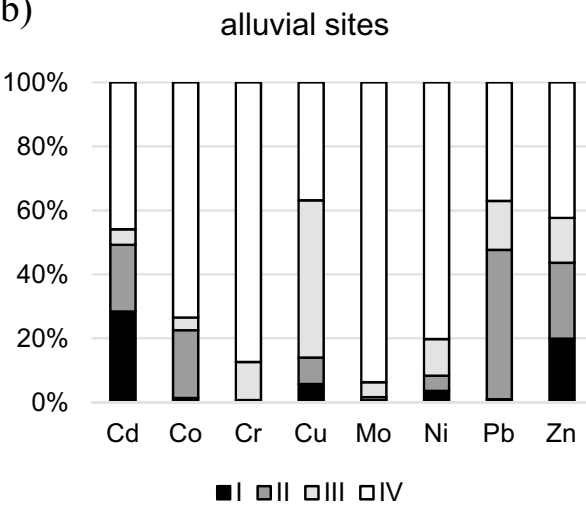

(c)

reference site

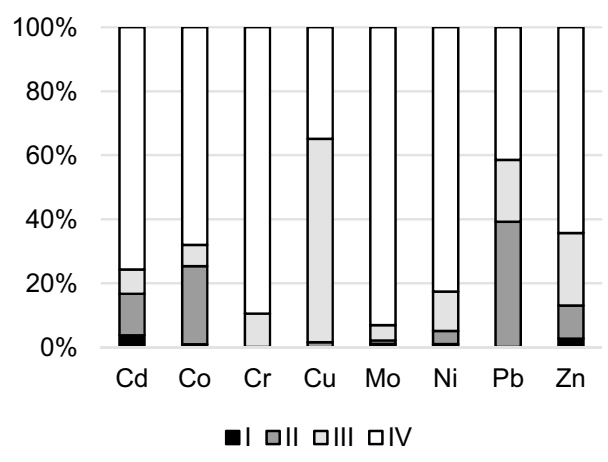

Figure 2. Distribution of potential toxic elements in 4 fractions in the soil depth of $0-10 \mathrm{~cm}$. I exchangeable and weak acid soluble fraction, $I I$ reducible fraction, $I I I$ oxidisable fraction, $I V$ residual fraction.

Relationships between HM fractions, soil properties, ecosystem types, and soil depth. The soil chemical and physical properties play an important role in the binding and migration of HMs in the soil. The correlation coefficient matrix for the HMs chemical fractions with the soil chemical and physical properties are shown in Tables 5 and 6.

Soil properties were selectively correlated with the HM fractions. The most numerous significant correlations with chemical properties occurred in the case of $\mathrm{Mo}$ (15), while the least occurred in the case of $\mathrm{Pb}(4)$. The most numerous significant correlations with physical properties occurred in the case of $\mathrm{Cu}(20)$ while the least occurred in the case of $\mathrm{Zn}(3)$.

The selected HM fractions of all HMs were significantly positively correlated with $\mathrm{pH}$ except for a significant negative correlation with the I $\mathrm{Cu}$ fraction. No statistically significant correlations were found between all HM fractions and Eh. The most numerous, mainly positive, correlations were found with the oxalate-extractable Fe and $\mathrm{Mn}$ in the case of Mo followed by $\mathrm{Cd}$, Co, and $\mathrm{Cr}$, while they did not occur in the case of Pb. Correlations with SOC occurred only with $\mathrm{Cu}$ (I and II fractions). Significantly positive correlations occurred between HA/ $\mathrm{FA}$ in the case of selected fractions of $\mathrm{Cr}, \mathrm{Mo}, \mathrm{Ni}, \mathrm{Pb}, \mathrm{Zn}$ and significantly negative only in the case of $\mathrm{Cu}$ ( $\mathrm{I}$ and II fractions) (Table 5).

The most numerous correlations were found with the different particle size fractions in the case of Ni followed by $\mathrm{Cr}, \mathrm{Cu}, \mathrm{Mo}$, and $\mathrm{Co}$, while they did not occur in the case of $\mathrm{Pb}$. While the selected $\mathrm{HM}$ fractions were mostly positively and significantly correlated with the content of clay particles, a reversed situation occurred in the case of the content of sand particles. No statistically significant correlations were found between all HM fractions and PD and only one negative correlation was found between BD and the IV Cd fraction. The water capacity parameters (MCWC and FWC), only negatively correlated with the selected HM fractions of Co, Cr, $\mathrm{Cu}, \mathrm{Ni}$, and $\mathrm{Pb}$. The $\mathrm{Cd}, \mathrm{Mo}$, and $\mathrm{Zn}$ fractions were not correlated with $\mathrm{MCWC}$ and $\mathrm{FWC}$. The most numerous correlations were found with the different porosity categories and MAC in the case of selected fractions of $\mathrm{Cu}$ followed by $\mathrm{Co}, \mathrm{Ni}$, and Cd (Table 6).

In Fig. 3, the hierarchical dendrogram of the linkage between the localities is shown for the surface soil layer (depth $0-10 \mathrm{~cm}$ ). The variables taken for this analysis are the same as the Pearson's correlation analysis.

The HCA has highlighted three large clusters, A, B, and C. Cluster A includes sites located in the lower part of the river located in the Podunajská pahorkatina Hills in the broader floodplains predominantly used as arable land. Cluster B includes two contaminated sites located in the Štiavnické vrchy Mountains and one site located in the lower part of the river situated in the Podunajská pahorkatina Hills in the broader floodplains used as arable land. The other localities are grouped in cluster C. Two clusters of lower hierarchical order can be recognised in 


\begin{tabular}{|c|c|c|c|c|c|c|c|}
\hline & & $\mathrm{pH} / \mathrm{KCl}$ & Eh & $\mathrm{Fe}_{\mathrm{o}}$ & $\mathrm{Mn}_{\mathrm{o}}$ & SOC & HA/FA \\
\hline \multirow{5}{*}{$\mathrm{Cd}$} & I & N.C & N.C & N.C & N.C & N.C & N.C \\
\hline & II & $0.472^{* \star}$ & N.C & $0.547^{* *}$ & $0.566^{\star *}$ & N.C & N.C \\
\hline & III & $0.464^{\star *}$ & N.C & $0.533^{\star *}$ & $0.410^{*}$ & N.C & N.C \\
\hline & IV & N.C & N.C & $-0.468^{\star *}$ & N.C & N.C & N.C \\
\hline & $\mathrm{T}$ & N.C & N.C & N.C & N.C & N.C & N.C \\
\hline \multirow{5}{*}{ Co } & I & N.C & N.C & N.C & $-0.362^{*}$ & N.C & N.C \\
\hline & II & N.C & N.C & $0.363^{*}$ & N.C & N.C & N.C \\
\hline & III & $0.373^{*}$ & N.C & $0.784^{* *}$ & $0.558^{* *}$ & N.C & N.C \\
\hline & IV & N.C & N.C & N.C & N.C & N.C & N.C \\
\hline & $\mathrm{T}$ & N.C & N.C & $0.423^{\star}$ & N.C & N.C & N.C \\
\hline \multirow{5}{*}{$\mathrm{Cr}$} & I & $0.537^{* *}$ & N.C & N.C & N.C & N.C & N.C \\
\hline & II & $0.476^{* *}$ & N.C & $0.413^{*}$ & N.C & N.C & N.C \\
\hline & III & $0.471^{\star *}$ & N.C & N.C & N.C & N.C & $0.396^{*}$ \\
\hline & IV & N.C & N.C & $-0.397^{*}$ & N.C & N.C & N.C \\
\hline & $\mathrm{T}$ & N.C & N.C & $-0.336^{*}$ & N.C & N.C & N.C \\
\hline \multirow{5}{*}{$\mathrm{Cu}$} & I & $-0.344^{*}$ & N.C & N.C & N.C & $-0.486^{* *}$ & $-0.430^{* x}$ \\
\hline & II & N.C & N.C & $0.445^{* *}$ & N.C & $-0.557^{* *}$ & $-0.354^{*}$ \\
\hline & III & N.C & N.C & $0.503^{* *}$ & N.C & N.C & N.C \\
\hline & IV & N.C & N.C & N.C & N.C & N.C & N.C \\
\hline & $\mathrm{T}$ & N.C & N.C & N.C & N.C & N.C & N.C \\
\hline \multirow{5}{*}{ Mo } & I & $0.336^{\star}$ & N.C & $0.464^{* *}$ & $0.368^{\star}$ & N.C & N.C \\
\hline & II & $0.461^{* *}$ & N.C & $0.573^{* *}$ & $0.794^{\star *}$ & N.C & $0.457^{* *}$ \\
\hline & III & $0.485^{\star *}$ & N.C & N.C & $0.457^{\star *}$ & N.C & N.C \\
\hline & IV & $0.499^{* *}$ & N.C & $0.494^{\star *}$ & $0.537^{\star *}$ & N.C & N.C \\
\hline & $\mathrm{T}$ & $0.508^{* *}$ & N.C & $0.493^{* *}$ & $0.548^{\star *}$ & N.C & N.C \\
\hline \multirow{5}{*}{$\mathrm{Ni}$} & I & N.C & N.C & N.C & N.C & N.C & N.C \\
\hline & II & $0.523^{\star *}$ & N.C & $0.386^{*}$ & $0.418^{*}$ & N.C & $0.353^{*}$ \\
\hline & III & $0.526^{* *}$ & N.C & N.C & N.C & N.C & $0.360^{*}$ \\
\hline & IV & N.C & N.C & N.C & N.C & N.C & N.C \\
\hline & $\mathrm{T}$ & $0.411^{\star}$ & N.C & N.C & N.C & N.C & N.C \\
\hline \multirow{5}{*}{$\mathrm{Pb}$} & I & N.C & N.C & N.C & N.C & N.C & N.C \\
\hline & II & N.C & N.C & N.C & N.C & N.C & N.C \\
\hline & III & $0.538^{* *}$ & N.C & N.C & N.C & N.C & $0.414^{\star}$ \\
\hline & IV & N.C & N.C & N.C & N.C & N.C & N.C \\
\hline & $\mathrm{T}$ & $0.368^{\star}$ & N.C & N.C & N.C & N.C & $0.373^{*}$ \\
\hline \multirow{5}{*}{$\mathrm{Zn}$} & I & $0.353^{*}$ & N.C & $0.431^{* *}$ & $0.346^{*}$ & N.C & N.C \\
\hline & II & $0.460^{* *}$ & N.C & N.C & N.C & N.C & N.C \\
\hline & III & $0.487^{* *}$ & N.C & N.C & N.C & N.C & $0.340^{*}$ \\
\hline & IV & N.C & N.C & N.C & N.C & N.C & N.C \\
\hline & $\mathrm{T}$ & $0.342^{\star}$ & N.C & N.C & N.C & N.C & N.C \\
\hline
\end{tabular}

Table 5. Pearson's correlation coefficient matrix of HMs in various fractions and soil chemical properties. $I$ exchangeable fraction, $I I$ reducible fraction, $I I I$ oxidisable fraction, $I V$ residual fraction, $T$ total content; ${ }^{*} r$ values shown in bold are significant at $\mathrm{p}<0.05 ;{ }^{* *} \mathrm{r}$ values shown in bold are significant at $\mathrm{p}<0.01, N . C$ no correlation.

C (C1 and C2). Cluster C1 includes three sites located in the middle part of the river located in the Štiavnické vrchy Mountains, of which one site is located within the riparian zone and two sites are used as grasslands. Cluster C2 includes predominantly sites located in the upper and middle part of the river located in the Štiavnické vrchy Mountains within the riparian zones or used as grasslands.

The ANOVA test was conducted to determine whether the differences in the content of the different HM fractions in the soils could be significant between the three ecosystem types (RZ, PG, AL) and the soil depths $(0-10,20-30 \mathrm{~cm})$

Based on the ANOVA results, significant differences emerged between the different ecosystem types for Cd, $\mathrm{Cr}$, and $\mathrm{Pb}$ in fractions I and $\mathrm{II}$, for $\mathrm{Cr}, \mathrm{Mo}, \mathrm{Ni}$, and $\mathrm{Pb}$ of fraction $\mathrm{IV}$, and for $\mathrm{Co}, \mathrm{Cr}, \mathrm{Mo}, \mathrm{Ni}$, and $\mathrm{Pb}$ of the total content. Within fraction III, the ecosystem types had an effect on all the observed HMs. The most significant differences between the different ecosystem types and the HM fractions were found in the case of $\mathrm{Cr}$, followed 


\begin{tabular}{|c|c|c|c|c|c|c|c|c|c|c|c|c|c|}
\hline & & \multicolumn{3}{|c|}{ Content of } & \multirow[b]{2}{*}{ PD } & \multirow[b]{2}{*}{ BD } & \multirow[b]{2}{*}{ MCWC } & \multirow[b]{2}{*}{ FWC } & \multirow[b]{2}{*}{ Pt } & \multirow[b]{2}{*}{ Pc } & \multirow[b]{2}{*}{ Pn } & \multirow[b]{2}{*}{ Ps } & \multirow[b]{2}{*}{ MAC } \\
\hline & & Clay & \begin{tabular}{|l|} 
Silt \\
\end{tabular} & Sand & & & & & & & & & \\
\hline \multirow{5}{*}{$\mathrm{Cd}$} & I & N.C & N.C & N.C & N.C & N.C & N.C & N.C & N.C & N.C & N.C & N.C & N.C \\
\hline & II & N.C & N.C & N.C & N.C & N.C & N.C & N.C & N.C & N.C & $-0.466^{\star}$ & N.C & $-0.431^{\star}$ \\
\hline & III & N.C & $-0.424^{\star}$ & N.C & N.C & N.C & N.C & N.C & N.C & N.C & N.C & N.C & N.C \\
\hline & IV & N.C & N.C & N.C & N.C & $-0.493^{* *}$ & N.C & N.C & $0.508^{* *}$ & N.C & $0.489^{* *}$ & N.C & $0.493^{* *}$ \\
\hline & $\mathrm{T}$ & N.C & N.C & N.C & N.C & N.C & N.C & N.C & N.C & N.C & N.C & N.C & N.C \\
\hline \multirow{5}{*}{ Co } & I & $0.509^{* *}$ & N.C & $-0.340^{*}$ & N.C & N.C & N.C & N.C & N.C & N.C & N.C & N.C & N.C \\
\hline & II & N.C & N.C & N.C & N.C & N.C & N.C & N.C & N.C & N.C & $-0.394^{\star}$ & N.C & N.C \\
\hline & III & N.C & N.C & N.C & N.C & N.C & N.C & N.C & N.C & N.C & $-0.421^{\star}$ & N.C & $-0.375^{\star}$ \\
\hline & IV & N.C & N.C & N.C & N.C & N.C & $-0.403^{*}$ & $-0.484^{* *}$ & N.C & $-0.484^{* *}$ & $0.495^{* *}$ & N.C & $0.494^{* *}$ \\
\hline & $\mathrm{T}$ & N.C & $-0.363^{\star}$ & $0.373^{\star}$ & N.C & N.C & N.C & $-0.430^{*}$ & N.C & $-0.430^{*}$ & N.C & $0.467^{\star}$ & N.C \\
\hline \multirow{5}{*}{$\mathrm{Cr}$} & I & N.C & N.C & N.C & N.C & N.C & N.C & N.C & N.C & N.C & N.C & N.C & N.C \\
\hline & II & N.C & N.C & N.C & N.C & N.C & N.C & N.C & N.C & N.C & N.C & N.C & N.C \\
\hline & III & $0.657^{* *}$ & N.C & \begin{tabular}{|l|l|}
$-0.445^{* *}$ \\
\end{tabular} & N.C & N.C & N.C & N.C & N.C & N.C & N.C & N.C & N.C \\
\hline & IV & $0.579^{* *}$ & $0.480^{* *}$ & $-0.611^{\star *}$ & N.C & N.C & $-0.473^{\star *}$ & N.C & N.C & N.C & N.C & $-0.550^{* *}$ & N.C \\
\hline & $\mathrm{T}$ & $0.626^{* *}$ & $0.464^{* *}$ & $-0.619^{\star \star}$ & N.C & N.C & $-0.433^{\star}$ & N.C & N.C & N.C & N.C & $-0.540^{\star *}$ & N.C \\
\hline \multirow{5}{*}{$\mathrm{Cu}$} & I & N.C & $-0.410^{*}$ & $0.421^{\star}$ & N.C & N.C & N.C & $-0.435^{\star}$ & $0.381^{*}$ & $-0.435^{\star}$ & $0.483^{* *}$ & $0.384^{\star}$ & $0.519^{* *}$ \\
\hline & II & N.C & $-0.533^{\star *}$ & $0.420^{*}$ & N.C & N.C & N.C & N.C & N.C & N.C & N.C & $0.527^{* *}$ & N.C \\
\hline & III & N.C & $-0.552^{\star *}$ & $0.496^{* *}$ & N.C & N.C & N.C & N.C & N.C & N.C & N.C & $0.428^{\star}$ & N.C \\
\hline & IV & N.C & N.C & N.C & N.C & N.C & N.C & N.C & $0.381^{*}$ & N.C & $0.544^{* *}$ & N.C & $0.542^{\star \star}$ \\
\hline & $\mathrm{T}$ & N.C & N.C & N.C & N.C & N.C & N.C & N.C & $0.389^{*}$ & N.C & $0.404^{*}$ & N.C & $0.438^{\star}$ \\
\hline \multirow{5}{*}{ Mo } & I & N.C & $-0.356^{*}$ & N.C & N.C & N.C & N.C & N.C & N.C & N.C & N.C & N.C & N.C \\
\hline & II & N.C & \begin{tabular}{|l|} 
N.C \\
\end{tabular} & N.C & N.C & N.C & N.C & N.C & N.C & N.C & N.C & N.C & N.C \\
\hline & III & N.C & N.C & N.C & N.C & N.C & N.C & N.C & N.C & N.C & N.C & N.C & N.C \\
\hline & IV & N.C & $-0.353^{\star}$ & $0.372^{\star}$ & N.C & N.C & N.C & N.C & N.C & N.C & N.C & N.C & N.C \\
\hline & $\mathrm{T}$ & N.C & $-0.345^{*}$ & $0.361^{\star}$ & N.C & N.C & N.C & N.C & N.C & N.C & N.C & N.C & N.C \\
\hline \multirow{5}{*}{$\mathrm{Ni}$} & I & N.C & N.C & N.C & N.C & N.C & N.C & N.C & N.C & N.C & N.C & N.C & N.C \\
\hline & II & $0.374^{*}$ & N.C & N.C & N.C & N.C & N.C & N.C & $-0.407^{\star}$ & N.C & $-0.486^{\star *}$ & N.C & $-0.467^{*}$ \\
\hline & III & $0.693^{* *}$ & N.C & $-0.509^{* *}$ & N.C & N.C & N.C & N.C & N.C & N.C & N.C & N.C & N.C \\
\hline & IV & $0.432^{* *}$ & $0.387^{*}$ & \begin{tabular}{|l|l|}
$-0.478^{* *}$ \\
\end{tabular} & N.C & N.C & $-0.486^{* *}$ & N.C & N.C & N.C & N.C & $-0.456^{*}$ & N.C \\
\hline & $\mathrm{T}$ & $0.573^{* *}$ & $0.353^{*}$ & $-0.511^{\star \star}$ & N.C & N.C & $-0.369^{\star}$ & N.C & N.C & N.C & N.C & $-0.447^{\star}$ & N.C \\
\hline \multirow{5}{*}{$\mathrm{Pb}$} & I & N.C & N.C & N.C & N.C & N.C & $-0.397^{*}$ & N.C & N.C & N.C & N.C & N.C & N.C \\
\hline & II & N.C & N.C & N.C & N.C & N.C & $-0.456^{* *}$ & $-0.413^{\star}$ & N.C & $-0.413^{*}$ & N.C & N.C & N.C \\
\hline & III & N.C & N.C & N.C & N.C & N.C & N.C & N.C & N.C & N.C & N.C & N.C & N.C \\
\hline & IV & N.C & N.C & N.C & N.C & N.C & N.C & N.C & N.C & N.C & N.C & N.C & N.C \\
\hline & $\mathrm{T}$ & N.C & N.C & N.C & N.C & N.C & N.C & N.C & N.C & N.C & N.C & N.C & N.C \\
\hline \multirow{5}{*}{$\mathrm{Zn}$} & I & $-0.424^{\star}$ & $-0.493^{* *}$ & $0.555^{\star *}$ & N.C & N.C & N.C & N.C & N.C & N.C & N.C & N.C & N.C \\
\hline & II & N.C & N.C & N.C & N.C & N.C & N.C & N.C & N.C & N.C & N.C & N.C & N.C \\
\hline & III & N.C & N.C & N.C & N.C & N.C & N.C & N.C & N.C & N.C & N.C & N.C & N.C \\
\hline & IV & N.C & N.C & N.C & N.C & N.C & N.C & N.C & N.C & N.C & N.C & N.C & N.C \\
\hline & $\mathrm{T}$ & N.C & N.C & N.C & N.C & N.C & N.C & N.C & N.C & N.C & N.C & N.C & N.C \\
\hline
\end{tabular}

Table 6. Pearson's correlation coefficient matrix of HMs in various fractions and soil physical properties. $I$ exchangeable fraction, $I I$ reducible fraction, $I I I$ oxidisable fraction, $I V$ residual fraction, $T$ total content, ${ }^{*} r$ values shown in bold are significant at $\mathrm{p}<0.05,{ }^{*} r$ values shown in bold are significant at $\mathrm{p}<0.01, N$.C no correlation.

by $\mathrm{Pb}, \mathrm{Cd}, \mathrm{Mo}, \mathrm{Ni} \mathrm{Co}, \mathrm{Cu}$, and $\mathrm{Zn}$ (Table 7). The effect of soil depth on HM fractions was revealed only in the case of $\mathrm{Cr}(\mathrm{I})$ and $\mathrm{Co}(\mathrm{III})$.

\section{Discussion}

The high content of HMs in different fractions in floodplain soils along the entire Štiavnica River watercourse proved to be a consequence of past mining activities. Many regions in Europe suffer from soil contamination caused by past mining activities ${ }^{24}$. On the other hand, in many developing countries, soil HM contamination has also become a severe problem due to rapid industrialization over the past several decades ${ }^{25}$.

Based on the findings, $\mathrm{Cd}, \mathrm{Cu}, \mathrm{Pb}$ and $\mathrm{Zn}$ are the most serious pollutants of the Štiavnica River floodplain. These metals are listed as priority control pollutants by the US Environmental Protection Agency due to their potential toxic, persistent, and irreversible characteristics. Therefore, their excessive accumulation in soils is of 


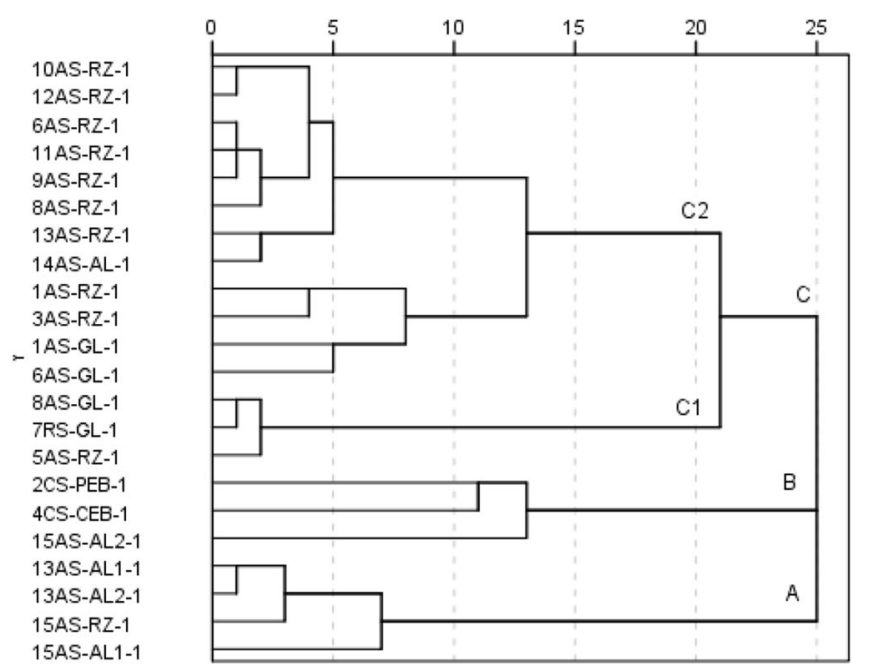

Figure 3. Dendrogram for hierarchical clustering of sites for the surface soil layer (Ward's method).

particular interest ${ }^{26}$. The total content of $\mathrm{Cd}, \mathrm{Cu}, \mathrm{Pb}$, and $\mathrm{Zn}$ in most localities significantly exceeded all limit values, including the Finnish guideline values. The Finnish standard values are considered a good approximation of the mean values of the different national systems in Europe and were used by Tóth et al. ${ }^{27}$ for the assessment of agricultural soils contamination in the EU. Contamination factors confirmed that the contamination by HMs at many AS localities was similar or even higher than at CS localities. The mine tailings contain residues characterised by a high content of HMs. The tailings were usually deposited untreated and left without improper management, being so unstable and prone to wind and water erosion ${ }^{28,29}$. HMs were generated during active mining in the eighteenth and nineteenth centuries and later released and transported by watercourse, and subsequently dispersed during floods along the entire Štiavnica River floodplain. This fact was revealed by HCA showing grouping localities on the basis of their similarities. Many of the study sites were under considerable ecological risk to the environment. Nevertheless, some of the localities located in the downstream part of the Podunajská pahorkatina Hills are still used intensively in agriculture.

The natural characteristics of the basin and the water course, including steepness and flow rate, have a significant impact on contamination, and they spread to the more distant surroundings. In the Štiavnické vrchy Mountains, the Štiavnica River flow is steep, narrow, and relatively shallow. In the downstream part of the basin in the Podunajská pahorkatina Hills, the Štiavnica River flow is milder, wider, and deeper. This situation supports heavy metal accumulation downstream, mainly in the part of the floodplain used for agriculture. In addition to the influence of anthropogenic activities, the higher content of clay particles could be the important environmental factor causing the absorption and adsorption of HM by soil. HM accumulated to toxic levels in agricultural land can potentially pose an environmental risk to other ecosystems and food production. Although the Štiavnica River is rather small and reflects the ecological conditions of its local environment and has an impact on the surrounding ecosystems and larger watercourses. Small rivers can have a big effect on larger water bodies and their surroundings ${ }^{30,31}$.

HM toxicity is related not only to the total content in the soil, but also to the distribution of its fractions. It is accepted that a large and relatively stable portion of HMs is present in the crystal lattice of minerals and the residual fraction. On the contrary, it is the mobile exchangeable fraction that can be used to evaluate the extent of environmental bioavailability. Among the HM fractions, the exchangeable fraction determines the most the actual environmental risk, and the reducible and oxidizable ones are the potentially bioavailable fractions. FES in floodplains is directly dependent on the capacity of soil structures to immobilize these mobile and/or potentially mobile and bioavailable fractions. Therefore, it is important to control the bioavailable fractions also for soil remediation $^{32}$. In terms of bioavailability, various species of metals are biologically more available than others. In our study, the content of $\mathrm{Cd}, \mathrm{Pb}$, and $\mathrm{Zn}$ in all, and $\mathrm{Cu}$ in most localities exceeded the Slovak threshold values for mobile forms of HMs in agricultural soils. This suggests that these elements can be readily available to plants and soil organisms ${ }^{33}$. Most HMs are toxic to humans, even at low contents.

The content of HMs in the residual fraction dominated at the RS locality. HMs bound in the residual fraction are naturally present in the parent rock. HMs of anthropogenic input tend to reside commonly in the first three fractions $\mathrm{s}^{34}$ and this is what we observed at the CS and AS localities in the case of $\mathrm{Cd}, \mathrm{Cu}, \mathrm{Pb}$, and $\mathrm{Zn}$. In the case of $\mathrm{Cd}$, the results indicated the relative importance of $\mathrm{Fe}-\mathrm{Mn}$ oxide bound, easily soluble, and exchangeable fractions for $\mathrm{Cd}^{35}$. The usual distribution of $\mathrm{Cd}$ in contaminated soils is exchangeable $>$ reducible $>$ oxidisable $>$ residual fraction ${ }^{36,37}$ what, in our study, was more similar to the HM fractionation at the CS localities. In the case of $\mathrm{Cu}$, we observed the great importance of organic matter bound, similar to what other authors have observed $^{26,38}$. In the case of $\mathrm{Pb}$, we found the relative importance of $\mathrm{Fe}$ and $\mathrm{Mn}$ oxide bound, in agreement with the findings of many authors ${ }^{39-41}$.

The correlation analysis allowed us to indicate the key factors that determine the binding of HMs in the soils and their mutual interactions. Many studies have dealt with the correlation relationships between HMs 


\begin{tabular}{|c|c|c|c|c|c|}
\hline & & \multicolumn{2}{|c|}{ Ecosystem type } & \multicolumn{2}{|c|}{ Soil depth } \\
\hline & & $F$-value & $P$-value & $F$-value & $P$-value \\
\hline \multirow{8}{*}{ I } & $\mathrm{Cd}$ & 5.313 & 0.005 & 0.068 & 0.795 \\
\hline & Co & 1.611 & 0.209 & 0.058 & 0.811 \\
\hline & $\mathrm{Cr}$ & 16.747 & 0.000 & 9.655 & 0.004 \\
\hline & $\mathrm{Cu}$ & 1.243 & 0.313 & 0.010 & 0.921 \\
\hline & Mo & 2.765 & 0.060 & 0.512 & 0.480 \\
\hline & $\mathrm{Ni}$ & 0.210 & 0.889 & 0.171 & 0.683 \\
\hline & $\mathrm{Pb}$ & 7.054 & 0.001 & 0.124 & 0.728 \\
\hline & $\mathrm{Zn}$ & 2.818 & 0.057 & 0.435 & 0.515 \\
\hline \multirow{8}{*}{ II } & $\mathrm{Cd}$ & 3.314 & 0.034 & 1.615 & 0.214 \\
\hline & Co & 1.927 & 0.148 & 0.032 & 0.859 \\
\hline & $\mathrm{Cr}$ & 16.518 & 0.000 & 0.800 & 0.379 \\
\hline & $\mathrm{Cu}$ & 1.809 & 0.168 & 0.133 & 0.718 \\
\hline & Mo & 1.607 & 0.210 & 1.010 & 0.324 \\
\hline & $\mathrm{Ni}$ & 1.931 & 0.147 & 0.341 & 0.564 \\
\hline & $\mathrm{Pb}$ & 12.993 & 0.000 & 0.689 & 0.414 \\
\hline & $\mathrm{Zn}$ & 1.605 & 0.211 & 0.050 & 0.825 \\
\hline \multirow{8}{*}{ III } & $\mathrm{Cd}$ & 5.131 & 0.006 & 0.205 & 0.655 \\
\hline & Co & 10.588 & 0.000 & 8.241 & 0.008 \\
\hline & $\mathrm{Cr}$ & 5.457 & 0.004 & 0.463 & 0.502 \\
\hline & $\mathrm{Cu}$ & 3.525 & 0.028 & 0.008 & 0.928 \\
\hline & Mo & 5.728 & 0.003 & 0.001 & 0.975 \\
\hline & $\mathrm{Ni}$ & 3.900 & 0.019 & 0.061 & 0.807 \\
\hline & $\mathrm{Pb}$ & 10.466 & 0.000 & 0.124 & 0.728 \\
\hline & $\mathrm{Zn}$ & 5.365 & 0.005 & 0.000 & 0.984 \\
\hline \multirow{8}{*}{ IV } & $\mathrm{Cd}$ & 1.002 & 0.407 & 5.065 & 0.032 \\
\hline & Co & 0.788 & 0.511 & 0.217 & \begin{tabular}{|l|}
0.645 \\
\end{tabular} \\
\hline & $\mathrm{Cr}$ & 5.564 & 0.004 & 0.064 & 0.802 \\
\hline & $\mathrm{Cu}$ & 0.531 & 0.665 & 2.511 & 0.124 \\
\hline & Mo & 5.790 & 0.003 & 0.271 & 0.607 \\
\hline & $\mathrm{Ni}$ & 4.084 & 0.016 & 0.348 & \begin{tabular}{|l|}
0.560 \\
\end{tabular} \\
\hline & $\mathrm{Pb}$ & 0.364 & 0.780 & 1.894 & \begin{tabular}{|l|}
0.180 \\
\end{tabular} \\
\hline & $\mathrm{Zn}$ & 1.745 & 0.181 & \begin{tabular}{|l|}
3.808 \\
\end{tabular} & 0.061 \\
\hline \multirow{8}{*}{$\mathrm{T}$} & $\mathrm{Cd}$ & 2.311 & 0.098 & 0.915 & 0.347 \\
\hline & Co & 3.975 & 0.018 & 0.020 & \begin{tabular}{|l|l|}
0.888 \\
\end{tabular} \\
\hline & $\mathrm{Cr}$ & 6.265 & 0.002 & 0.021 & 0.885 \\
\hline & $\mathrm{Cu}$ & 1.621 & 0.207 & 0.876 & \begin{tabular}{|l|}
0.357 \\
\end{tabular} \\
\hline & Mo & 5.912 & 0.003 & 0.214 & \begin{tabular}{|l|}
0.647 \\
\end{tabular} \\
\hline & $\mathrm{Ni}$ & 6.855 & 0.001 & 0.137 & \begin{tabular}{|l|}
0.714 \\
\end{tabular} \\
\hline & $\mathrm{Pb}$ & 4.175 & 0.015 & 1.435 & 0.241 \\
\hline & $\mathrm{Zn}$ & 1.891 & 0.154 & 1.490 & 0.232 \\
\hline
\end{tabular}

Table 7. Effect of ecosystem type and soil depth on heavy metal fractions. The P-values highlighted in bold are highly statistically significant $(\mathrm{P}<0.001)$ and statistically significant $(\mathrm{P}<0.05)$.

and physicochemical properties ${ }^{42-44}$. However, the results obtained are not unequivocal ${ }^{45}$ but can still serve as a valuable source of information for the management of contaminated sites.

Although soil $\mathrm{pH}$ is considered to affect the chemical composition of soil solution and also the bioavailability of heavy metals ${ }^{46,47}$, we found also no correlations between the I HM fraction and the $\mathrm{pH}$ in the case of Cd, Co, $\mathrm{Ni}$ and $\mathrm{Pb}$. Furthermore, in our study, no correlations with Eh were found, although heavy metal behaviour is strongly dependent on redox gradients ${ }^{46}$. Fe and $\mathrm{Mn}$ can strongly adsorb divalent HM cations, and their positive correlation with HMs is more prevalent in the studies ${ }^{48}$ similar to our study. Negative correlation is less common $^{49}$.

In our study, we confirmed the importance of the content of clay and silt particles as well as SOC as sorbents with great absorptive capacity that support FES. This is consistent with the findings of other authors ${ }^{50}$ who claimed that SOC and clay contents ${ }^{51}$ have substantial influences on the variability of the HMs. The grain size is one of the essential factors influencing $\mathrm{HM}$ contents in soil ${ }^{52}$. The I fraction of $\mathrm{Cu}, \mathrm{Mo}$, and $\mathrm{Zn}$ is negatively correlated with the content of clay or silt particles, and the I fraction of $\mathrm{Cu}$ is negatively correlated with SOC. Thus, a higher clay and silt content can immobilize metals, such as $\mathrm{Cu}, \mathrm{Mo}$, and $\mathrm{Zn}$, and a higher organic content 
can immobilize metals such as $\mathrm{Cu}$. This finding confirmed the importance of organic soil amendments used to immobilize HMs in soils by changing the speciation from initially highly bioavailable forms to much less bioavailable fractions associated with organic matter ${ }^{53}$. Organic matter is known to have significant immobilizing effects on HMs including $\mathrm{Cu}^{54,55}$. Therefore, the retention of $\mathrm{HM}$ induced by organic amendment is, on the whole, mainly attributed to an increase in surface charges ${ }^{56}$ and to FES support.

HM interactions with soil hydro-physical properties are not observed and evaluated so often. But these interactions exist and even poor physical properties such as lower soil porosity, and reduced water holding capacity may indirectly result from HM contamination ${ }^{57}$. Zhang et al. ${ }^{44}$ similarly to our results, consider soil porosity as one of the dominant factors determining the distribution of HMs and their fractions in the micropore-dominated riparian soil.

We recorded significant differences between different ecosystem types for all HMs in different fractions but mainly for $\mathrm{Cr}$ and $\mathrm{Pb}$, and no effect of soil depth on $\mathrm{HM}$ fractions was revealed, except for $\mathrm{Cr}$ (I) and $\mathrm{Co}$ (III). This indicates that different types of ecosystem and land-use can influence HMs and their fraction content in soils, as documented by many authors ${ }^{58,59}$.

\section{Conclusions}

Based on our findings, there is a significant degree of $\mathrm{HM}$ contamination, particularly $\mathrm{Cd}, \mathrm{Cu}, \mathrm{Pb}$, and $\mathrm{Zn}$ in the floodplain soils around the Štiavnica River. This pollution originated mainly from past mining activities. The entire downstream transport of suspended solids was a source of HMs for distant soils and, furthermore, pollution caused serious contamination to distant agricultural land in Podunajská pahorkatina Hills.

We comprehensively analysed soil properties that affect HM fractionation and bioavailability. The distribution of HMs in the different fractions, mainly in exchangeable ones, suggested that the mobility and bioavailability of the metals probably declined to the order of $\mathrm{Cd}, \mathrm{Zn}, \mathrm{Cu}, \mathrm{Ni}$, and $\mathrm{Pb}$. The dominance or significant portion of HM in the exchangeable fraction indicates a lower or higher FES capacity provided by floodplain soils, also determined by other soil properties. Soil properties were selectively correlated with the HM fractions. Based on the ANOVA results, the effect of different ecosystem types on the HM fractions was revealed. Thus, soil properties and ecosystem types confirmed a special role in the fate of heavy metals.

These results highlight the importance of considering the HM fractionation as well as the soil properties of different ecosystem types, when making strategic decisions to arrange anthropogenic activities and prevent risk to the environment and human health. There is an urgent need to preserve existing floodplains as natural resources and begin to restore them. This study could help managers adopt a more ecosystem-based approach to floodplain management by helping to reduce pollution. Furthermore, we should consider the adverse influences on ecosystems, living organisms, and public health.

Received: 15 October 2021; Accepted: 3 February 2022

Published online: 21 February 2022

\section{References}

1. EEA. Floodplains: A natural system to preserve and restore. EEA report No. 24/2019. ISBN 978-92-9480-211-8, ISSN 1977-8449. https://doi.org/10.2800/431107 (2019).

2. De Groot, R. et al. Global estimates of the value of ecosystems and their services in monetary units. Ecosyst. Serv. 1, 50-61. https:// doi.org/10.1016/j.ecoser.2012.07.005 (2012).

3. Costanza, R. et al. Changes in the global value of ecosystem services. Glob. Environ. Chang. 26, 152-158. https://doi.org/10.1016/j. gloenvcha.2014.04.002 (2014).

4. Tockner, K. \& Stanford, J. A. Riverine flood plains: Present state and future trends. Environ. Conserv. 29(03), 308-330. https://doi. org/10.1017/S037689290200022X (2002).

5. Panagos, P., Van Liedekerke, M., Yigini, Y. \& Montanarella, L. Contaminated sites in Europe: Review of the current situation based on data collected through a European network. J. Environ. Public Health 2013, 158764. https://doi.org/10.1155/2013/158764 (2013).

6. Wan, X., Dong, H., Feng, L., Lin, Z. \& Luo, Q. Comparison of three sequential extraction procedures for arsenic fractionation in highly polluted sites. Chemosphere 178, 402-410. https://doi.org/10.1016/j.chemosphere.2017.03.078 (2017).

7. Ahn, Y. et al. Heavy metal speciation with prediction model for heavy metal mobility and risk assessment in mine-affected soils. Environ. Sci. Pollut. Res. 27, 3213-3223. https://doi.org/10.1007/s11356-019-06922-0 (2019).

8. Mesut, A. \& Kara, D. Assessment of sequential extraction methods for the prediction of bioavailability of elements in plants grown on agricultural soils near to boron mines in Turkey. Talanta 200, 41-50. https://doi.org/10.1016/j.talanta.2019.03.031 (2019).

9. Reyes, A. et al. Source patterns of potentially toxic elements (PTEs) and mining activity contamination level in soils of Taltal city (northern Chile). Environ. Geochem. Health 42, 2573-2594. https://doi.org/10.1007/s10653-019-00404-5 (2019).

10. Macklin, M. G. et al. A geomorphological approach to the management of rivers contaminated by metal mining. Geomorphology 79, 423-447. https://doi.org/10.1016/j.geomorph.2006.06.024 (2006).

11. Mayes, W. M., Potter, H. A. B. \& Jarvis, A. P. Riverine flux of metals from historically mined ore fields in England and Wales. Water Air. Soil. Pollut. 224, 1425. https://doi.org/10.1007/s11270-012-1425-9 (2013).

12. Lamine, S. et al. Heavy metal soil contamination detection using combined geochemistry and field spectroradiometry in the United Kingdom. Sensors 19, 762. https://doi.org/10.3390/s19040762 (2019).

13. Opperman, J., Moyle, P., Larsen, E., Florsheim, J., Manfree, A. Ecosystem services and floodplain reconciliation in Floodplains: Processes and Management for Ecosystem Services. 99-114 (University of California Press, 2017). https://doi.org/10.1525/97805 20966321-011

14. Foulds, S. A. et al. Flood-related contamination in catchments affected by historical metal mining: An unexpected and emerging hazard of climate change. Sci. Total Environ. 476, 165-180. https://doi.org/10.1016/j.scitotenv.2013.12.079 (2014).

15. Frohne, T., Rinklebe, J. \& Diaz-Bone, R. A. Contamination of floodplain soils along the Wupper river, Germany, with As Co, Cu, $\mathrm{Ni}, \mathrm{Sb}$, and $\mathrm{Zn}$ and the impact of predefinite redox variations on the mobility of these elements. Soil Sediment Contam. Int. J. 23(7), 779-799. https://doi.org/10.1080/15320383.2014.872597 (2014).

16. Pan, L. et al. Potentially toxic element pollution levels and risk assessment of soils and sediments in the upstream river, Miyun reservoir, China. Int. J. Environ. Res. Public Health 15, 2364. https://doi.org/10.3390/ijerph15112364 (2018). 
17. Wang, J. et al. Towards a systematic method for assessing the impact of chemical pollution on ecosystem services of water systems. J. Environ. Manage. 281, 111873. https://doi.org/10.1016/j.jenvman.2020.111873 (2021).

18. Kanat, G., Ikzoglu, B., Erguven, G. O. \& Akgun, B. Determination of pollution and heavy metal fractions in golden horn sediment sludge (Istanbul, Turkey). Pol. J. Environ. Stud. 27, 2605-2611. https://doi.org/10.15244/pjoes/80805 (2018).

19. Rauret, G. et al. Improvement of the BCR three step sequential extraction procedure prior to certification of new sediment and soil reference materials. J. Environ. Monit. 1, 57-61. https://doi.org/10.1039/A807854H (1999).

20. Pansu, M. \& Gautheyrou, J. Handbook of soil analysis. Mineralogical organic and inorganic methods (Springer, 2006).

21. Varol, M. Assessment of heavy metal contamination in sediments of the Tigris river (Turkey) using pollution indices and multivariate statistical techniques. J. Hazard. Mater. 195, 355-364. https://doi.org/10.1016/j.jhazmat.2011.08.051 (2011).

22. Tomlinson, D., Wilson, J., Harris, C. \& Jeffrey, D. Problems in the assessment of heavy-metal levels in estuaries and the formation of a pollution index. Helgoländer Meeresuntersuchungen 33(1-4), 566-575. https://doi.org/10.1007/BF02414780 (1980).

23. Hakanson, L. An ecological risk index for aquatic pollution control: A sedimentological approach. Water Res. 14(8), 975-1001. https://doi.org/10.1016/0043-1354(80)90143-8 (1980).

24. Pelica, J. et al. The paradigm of high concentration of metals of natural or anthropogenic origin in soils: The case of Neves-Corvo mine area (Southern Portugal). J. Geochem. Explor. 186, 12-23. https://doi.org/10.1016/j.gexplo.2017.11.021 (2018).

25. Li, Z., Ma, Z., Kuijp, T. J. V. D., Yuan, Z. \& Huang, L. A review of soil heavy metal pollution from mines in China: Pollution and health risk assessment. Sci. Total Environ. 468-469, 843-853. https://doi.org/10.1016/j.scitotenv.2013.08.090 (2014).

26. Yang, B., Ren, J., Wang, M., Luo, H. \& Cao, Y. Concentrations and chemical fractions of $\mathrm{Cu}, \mathrm{Zn}, \mathrm{Cd}$, and $\mathrm{Pb}$ at ten metallurgical sites in China. Environ. Sci. Pollut. Res. 26, 3603-3611. https://doi.org/10.1007/s11356-018-3881-2 (2019).

27. Tóth, G., Hermann, T., Da Silva, M. R. \& Montanarella, L. Heavy metals in agricultural soils of the European Union with implications for food safety. Environ. Int. 88, 299-309. https://doi.org/10.1016/j.envint2015.12.017 (2016).

28. Shukurov, N. et al. Coupling geochemical, mineralogical and microbiological approaches to assess the health of contaminated soil around the Almalyk mining and smelter complex, Uzbekistan. Sci. Total Environ. 476-477, 447-459. https://doi.org/10.1016/j. scitotenv.2014.01.031 (2014).

29. Christou, A., Theologides, Ch. P., Costa, C., Kalavrouziotis, I. K. \& Varnavas, S. P. Assessment of toxic heavy metals concentrations in soils and wild and cultivated plant species in Limni abandoned copper mining site, Cyprus. J. Geochem. Explor. 178, 16-22. https://doi.org/10.1016/j.gexplo.2017.03.012 (2017).

30. Pimentel, D. et al. Water resources: Agricultural and environmental issues. BioSciences 54, 900-918. https://doi.org/10.1641/0006568(2004)054[0909:WRAAEI]2.0.CO;2 (2004).

31. Yurova, Y. \& Shirokova, V. Geological assessment of anthropogenic impacts on the Osetr river basin. Geosciences 10, 121. https:// doi.org/10.3390/geosciences10040121 (2020).

32. He, Y., Lin, H., Jin, X., Dong, Y. \& Luo, M. Simultaneous reduction of arsenic and cadmium bioavailability in agriculture soil and their accumulation in Brassica chinensis L. by using minerals. Ecotoxicol. Environ. Saf. 198, 110660. https://doi.org/10.1016/j. ecoenv.2020.110660 (2020).

33. Qasim, B. \& Motelica-Heino, M. Potentially toxic element fractionation in technosoils using two sequential extraction schemes. Environ. Sci. Pollut. Res. 21(7), 5054-5065. https://doi.org/10.1007/s11356-013-2457-4 (2014).

34. Ratuzny, T., Gong, Z. \& Wilke, B. M. Total concentrations and speciations of heavy metals in soils of the Shenyang Zhangshi Irrigation Area, China. Environ. Monit. Assess. 156(1-4), 171-180. https://doi.org/10.1007/s10661-008-0473-5 (2009).

35. Alan, M. \& Kara, D. Comparison of a new sequential extraction method and the BCR sequential extraction method for mobility assessment of elements around boron mines in Turkey. Talanta 194, 189-198. https://doi.org/10.1016/j.talanta.2018.10.030 (2019).

36. Kaasalainen, M. \& Yli-Halla, M. Use of sequential extraction to assess metal partitioning in soils. Environ. Pollut. 126, 225-233. https://doi.org/10.1016/S0269-7491(03)00191-X (2003).

37. Khadhar, S., Sdiri, A., Chekirben, A., Azouzi, R. \& Charef, A. Integration of sequential extraction, chemical analysis and statistical tools for the availability risk assessment of heavy metals in sludge amended soils. Environ. Pollut. https://doi.org/10.1016/j.envpol. 2020.114543 (2020).

38. Gabarrón, M. et al. Effect of land use and soil properties in the feasibility of two sequential extraction procedures for metals fractionation. Chemosphere 218, 266-272. https://doi.org/10.1016/j.chemosphere.2018.11.114 (2019).

39. Zhang, G. et al. Heavy metal fractions and ecological risk assessment in sediments from urban, rural and reclamation-affected rivers of the Pearl River Estuary, China. Chemosphere 184, 278-288. https://doi.org/10.1016/j.chemosphere.2017.05.155 (2017).

40. Carvalho Aguiar, V. M., Abuchaera, P. F. F., Neto, J. A. B. \& Oliveira, A. S. Environmental assessment concerning trace metals and ecological risk at Guanabara Bay, RJ Brazil. Environ. Monit. Assess. 190, 448. https://doi.org/10.1007/s10661-018-6833-x (2018).

41. Ates, A., Demirel, H. \& Mergul, N. Risk assessment and chemical fractionation of heavy metals by BCR sequential extraction in soil of the Sapanca Lake Basin, Turkey. Polish J. Environ. Stud. 29, 1523-1533. https://doi.org/10.15244/pjoes/101609 (2020).

42. Zhang, Q. et al. Distribution and contamination assessment of soil heavy metals in the Jiulongiiang river catchment, southeast China. Int. J. Environ. Res. Public Health 16(23), 4674. https://doi.org/10.3390/ijerph16234674 (2019).

43. Salem, M. A., Bedade, D. K., Al-Ethawi, L. \& Al-Waleed, S. M. Assessment of physicochemical properties and concentration of heavy metals in agricultural soils fertilized with chemical fertilizers. Heliyon 6(10), e05224. https://doi.org/10.1016/j.heliyon.2020. e05224 (2020).

44. Zhang, W. et al. Reservoir operation-induced hydrodynamic disturbances affect the distributions of $\mathrm{Cd}, \mathrm{Cu}$, and $\mathrm{Pb}$ in the riparian soil of the water-level-fluctuation zone. J. Soils Sediments 21, 2343-2356. https://doi.org/10.1007/s11368-021-02927-w (2021).

45. Zglobicki, W., Telecka, M. \& Pasierbinska, A. Impact of physicochemical characteristics of colluvial and alluvial soils on $\mathrm{Cd}, \mathrm{Cu}$, $\mathrm{Pb}$ and $\mathrm{Zn}$ content (east Poland). Polish J. Soils Sci. 48(2), 213-228. https://doi.org/10.17951/pjss.2015.48.2.213 (2015).

46. Zhang, J. et al. Bioavailability and soil-to-crop transfer of heavy metals in farmland soils: A case study in the Pearl River Delta, South China. Environ. Pollut. 235, 710-719. https://doi.org/10.1016/j.envpol.2017.12.106 (2018).

47. Wei, B. et al. The availability and accumulation of heavy metals in greenhouse soils associated with intensive fertilizer application. Int. J. Environ. Res. Public Health 17, 5359. https://doi.org/10.3390/ijerph17155359 (2020).

48. Shan, Y. et al. Identification of sources of heavy metal in agricultural soils using multivariate analysis and GIS. J. Soils Sediments 13, 720-729. https://doi.org/10.1007/s11368-012-0637-3 (2013).

49. Hou, D., O'Connor, D., Nathanail, P., Tian, L. \& Ma, Y. Integrated GIS and multivariate statistical analysis for regional scale assessment of heavy metal soil contamination: A critical review. Environ. Pollut. 231, 1188-1200. https://doi.org/10.1016/j.envpol.2017. $07.021(2017)$.

50. Ayoubi, S., Jabbari, M. \& Khadem, H. Multiple linear modelling between soil properties, magnetic susceptibility and heavy metals in various land uses. Model. Earth Syst. Environ. 4, 579-589. https://doi.org/10.1007/s40808-018-0442-0 (2018).

51. Ou, J. et al. In situ immobilization of toxic metals in soil using Maifan stone and illite/smectite clay. Sci. Rep. 8, 4618. https://doi. org/10.1038/s41598-018-22901-w (2018).

52. Jung, M. C. Heavy metal concentrations in soils and factors affecting metal uptake by plants in the vicinity of a Korean Cu-W mine. Sensors 8, 2416-2423. https://doi.org/10.3390/s8042413 (2008).

53. Walker, D. J., Clemente, R. \& Bernal, M. P. Contrasting effects of manure and compost on soil pH, heavy metal availability and growth of Chenopodium album L. in a soil contaminated by pyritic mine waste. Chemosphere 57, 215-224. https://doi.org/10. 1016/j.chemosphere.2004.05.020 (2004). 
54. Van Herwijnen, R. et al. Remediation of metal contaminated soil with mineral-amended composts. Environ. Pollut. 150, 347-354. https://doi.org/10.1016/j.envpol.2007.01.023 (2007).

55. Alvarenga, P. et al. Organic residues as immobilizing agents in aided phytostabilization: (I) Effects on soil chemical characteristics. Chemosphere 74, 1292-1300. https://doi.org/10.1016/j.chemosphere.2008.11.063 (2009).

56. Clark, G. J., Dodgshun, N., Sale, P. W. G. \& Tang, C. Changes in chemical and biological properties of a sodic clay subsoil with addition of organic amendments. Soil Biol. Biochem. 39, 2806-2817. https://doi.org/10.1016/j.soilbio.2007.06.003 (2007).

57. Lwin, C. S., Seo, B., Kim, H., Owens, G. \& Kim, K. Application of soil amendments to contaminated soils for heavy metal immobilization and improved soil quality: A critical review. Soil Sci. Plant Nutr. 64(2), 156-167. https://doi.org/10.1080/00380768.2018. 1440938 (2018).

58. Yan, X., Liu, M., Zhong, J., Guo, J. \& Wu, W. How human activities affect heavy metal contamination of soil and sediment in a long-term reclaimed area of the Liaohe river delta, North china. Sustainability 10, 338. https://doi.org/10.3390/su10020338 (2018).

59. Huang, L. et al. Heavy metals distribution, source and ecological risk assessment in Huixian wetland, south China. Water 12, 431. https://doi.org/10.3390/w12020431 (2019).

\section{Acknowledgements}

This work was supported by the Slovak Scientific Grant Agency_VEGA under the contract no. VEGA 1/0184/21. The research of abiotic soil parameters was done by the equipment supported by Operational Programme Research and Development under the contract no. ITMS- 26210120024. The authors would like to acknowledge the support received from professor Michael Komárek (the Czech University of Life Sciences Prague) for facilitating the access to the laboratory and his professional support. The authors appreciate the effort of the laboratory engineer Marie Králová assisting the geochemistry analyses.

\section{Author contributions}

Conceptualization: R.K.; Methodology: R.K.; Formal analysis and investigation: R.K., J.V., N.B., M.K., L.J.; Writing_original draft preparation: R.K.; Writing_review and editing: J.V., N.B., M.K., L.J.; Funding acquisition: R.K.; Supervision: R.K.

\section{Funding}

This work was supported by the Scientific Grant Agency of the Ministry of Education, Science, Research and Sport of the Slovak Republic (VEGA No. 1/0184/21).

\section{Competing interests}

The authors declare no competing interests.

\section{Additional information}

Correspondence and requests for materials should be addressed to R.K.

Reprints and permissions information is available at www.nature.com/reprints.

Publisher's note Springer Nature remains neutral with regard to jurisdictional claims in published maps and institutional affiliations.

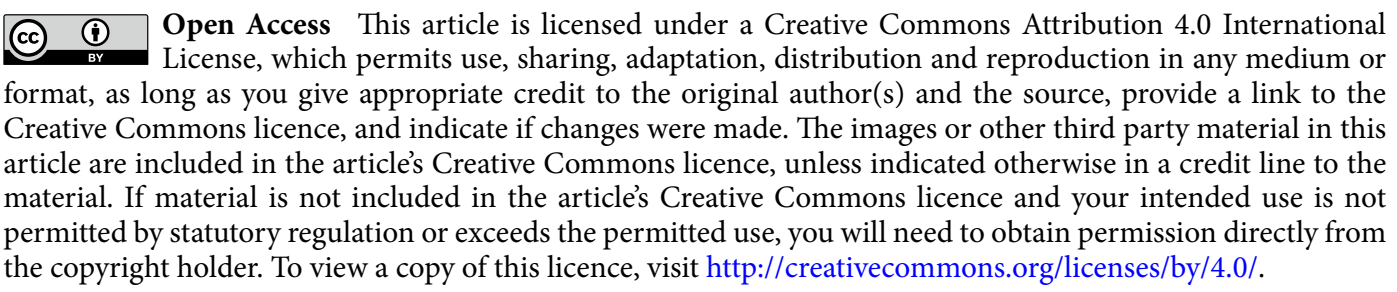

(C) The Author(s) 2022 